\title{
Influence des potentiels d'interaction sur les propriétés de transport des plasmas thermiques : exemple d'application le plasma argon hydrogène à la pression atmosphérique
}

\author{
J. Aubreton et P. Fauchais \\ Equipe Thermodynamique, Céramiques Nouvelles, LA 320, \\ Université de Limoges, 123, Avenue Albert Thomas, 87060 Limoges Cedex, France
}

(Reçu le 31 mars 1982, révisé le 27 septembre 1982, accepté le 4 octobre 1982)

\begin{abstract}
Résumé. - Après un rappel sur le calcul de la composition du plasma et sur la méthode d'évaluation des propriétés de transport, nous consacrons un paragraphe aux formes du potentiel d'interaction entre particules (atomes, ions, molécules) les plus souvent utilisées dans la littérature et nous indiquons celles pour lesquelles des tables permettent l'obtention des intégrales de collision.

La deuxième partie porte sur l'application de ces calculs aux mélanges argon-hydrogène pour lesquels nous sélectionnons, dans la littérature, les potentiels d'interactions ou les sections efficaces de transfert nécessaires à la détermination des intégrales de collision. La composition du plasma est calculée par minimisation de l'énergie libre de Gibbs à l'équilibre thermodynamique complet, à la pression atmosphérique et pour une température variant de $500 \mathrm{~K}$ à $15000 \mathrm{~K}$. Les propriétés de transport (viscosité, conductibilité thermique et conductivité électrique) sont alors obtenues pour cinq valeurs du rapport $\mathrm{H}_{2} /\left(\mathrm{H}_{2}+\mathrm{Ar}\right)$ : de l'hydrogène pur à l'argon pur par saut de $25 \%$. Les données (intégrales de collision et fonctions de partition) sont introduites dans le programme sous forme de polynomes.
\end{abstract}

Finalement, nos résultats sont comparés avec ceux de la littérature.

\begin{abstract}
After a brief presentation of the calculation methods of the transport properties for a thermal plasma at atmospheric pressure, we present in the first part of this paper the most frequently used and most recent expressions of the interaction potentials between the different types of particles (atoms, ions, molecules) and we indicate those with calculated collision integral tables. The second part deals with the application of such calculations to the argon-hydrogen mixture. The collision integrals are determined from selected interaction potentials or from transfer cross-sections. The plasma composition (in LTE) is calculated at atmospheric pressure by the free energy minimization procedure and for temperatures varying from $500 \mathrm{~K}$ to $15000 \mathrm{~K}$. The transport properties (dynamic viscosity, thermal and electrical conductivity) are calculated for five values of the $\mathrm{H}_{2} /\left(\mathrm{H}_{2}+\mathrm{Ar}\right)$ ratio : from pure hydrogen to pure argon by $25 \%$ incrementation. In the computer program, data (collision integrals and partition functions) have been expressed as polynomial forms. At last, our results are compared to those of the literature.
\end{abstract}

Introduction - L'utilisation des plasmas thermiques dans l'industrie est restée, pendant longtemps (pratiquement de 1965 à 1975), limitée au découpage [1], à la soudure [2] et à la projection de revêtements protecteurs réfractaires ou non [3, 4]. Cependant 1975 voit le début de nombreuses études à des échelles pilotes (jusqu'à $1 \mathrm{MW}$ ) de réacteurs à plasma destinés à des opérations de métallurgie extractive [5-8] et à la production de gaz réducteurs ou oxydants à haute température $[2,9]$ et les années $80-81$ concrétisent ces recherches avec la mise en service d'unités de production dans lesquelles les plasmas thermiques jouent un rôle prépondérant : production de fonte par Cokerill
(Belgique) [10] et par S.K.F. (Suède) [11], d'éponge de fer par S.K.F. [12], production d'acier par Foster Wheeler (Grande-Bretagne) [8], affinage des aciers par Daido(Japon) [13]. De nombreuses autres applications sont égaiement envisagées : métallurgie extractive du molybdène [14], récupération et refusion de métaux exotiques [15], aciers surnitrurés...

Toutes ces opérations dépendent, entre autres, de l'aptitude des plasmas à améliorer considérablement, notamment pour des gaz di- ou polyatomique, les transferts thermiques [16]. Dans cette optique le calcul des propriétés de transport des plasmas est capital et c'est donc pourquoi le sous-comité Chimie des 
Plasmas de l'I.U.P.A.C. a entrepris une bibliographie exhaustive des études faites sur ces propriétés [17]. Celle-ci montre l'existence d'une très grande disparité entre les valeurs de ces propriétés même pour des gaz simples comme l'argon, l'hydrogène, l'azote..., disparités dues, pour les résultats théoriques, d'une part à l'ordre d'approximation retenu pour le calcul des propriétés de transport lors de la résolution de l'équation de Boltzmann et d'autre part aux valeurs des potentiels d'interaction utilisés pour le calcul des intégrales de collision [18]. Il convient également de noter que, mis à part quelques calculs récents $[19,20]$ notamment pour des mélanges complexes avec des vapeurs métalliques, la grande majorité des calculs sur des propriétés de transport est antérieure à 1976 [17]. Compte tenu des progrès actuels de la physique atomique dans l'évaluation expérimentale ou théorique par des méthodes ab initio, des données relatives aux potentiels d'interaction, il nous a paru bon de reprendre le calcul de ces propriétés de transport dans les plasmas thermiques (à la pression atmosphérique) des mélanges $\mathrm{Ar}-\mathrm{H}_{2}$ qui sont parmi les plus utilisés tant en projection qu'en four de refusion ou de métallurgie.

Après un rappel sur le calcul de la composition et sur la méthode d'évaluation des propriétés de transport, nous consacrerons un paragraphe aux formes analytiques du potentiel d'interaction et nous indiquerons ceux pour lesquels des tables permettent l'obtention des intégrales de collision. La deuxième partie portera sur l'application de ces calculs aux mélanges argonhydrogène : calcul des intégrales de collision à partir des potentiels d'interaction ou des sections efficaces de transfert et brève présentation de nos résultats pour un plasma à l'équilibre thermodynamique et à la pression atmosphérique (pour une température évoluant de $500 \mathrm{~K}$ à $15000 \mathrm{~K}$, composition et propriétés de transport). Nous nous sommes limités à cette gamme de températures qui est suffisante pour les modélisations dans les plasmas d'arc d'autant que pour des températures supérieures les collisions ont uniquement lieu entre particules chargées et dépendent du seul potentiel de Coulomb avec écran, ce qui fait que les propriétés de transport deviennent simples à déterminer si l'on dispose de la composition du plasma.

1. Principes généraux. - 1.1 CAlCUl de La COMPOSITION. - Le calcul de la composition se fait à l'équilibre, ce qui permet la détermination des grandeurs thermodynamiques à partir des fonctions de partition et l'utilisation de la loi de Saha pour le calcul de la composition en présence d'espèces chargées par une méthode, désormais classique, de minimisation de l'énergie libre à l'aide de multiplicateurs de Lagrange [21].

Aux basses températures le plasma contient très peu d'espèces ionisées, on le considère comme un gaz parfait en supposant qu'il n'y a aucune interaction entre les particules (potentiel à court rayon d'action) en dehors des collisions nécessaires à l'établissement de l'équilibre. L'énergie libre s'écrit alors :

$$
G=\sum_{i} N_{i} \mu_{i}
$$

où $N_{i}$ et $\mu_{i}$ sont, respectivement, le nombre de particules et le potentiel chimique de gaz parfait de l'espèce $i$.

Cependant avec l'augmentation de la température, apparait le problème du potentiel d'interaction de type coulombien (à long rayon d'action) entre particules chargées qui fait naître une énergie d'interaction, en plus de l'énergie d'agitation thermique. Pour la déterminer, nous utilisons l'approximation de DebyeHuckel (dans un plasma à la pression atmosphérique le critère de Griem [22] :

$$
\sum_{z \neq 0} N_{z} \geqslant\left(8 \pi d^{3} / V\right)^{-1}
$$

est toujours vérifié et l'énergie de Coulomb est extrêmement faible par rapport à l'énergie thermique). Soit donc pour l'énergie libre :

$$
G=\sum_{i} N_{i} \mu_{i}-\frac{k T V}{8 \pi d^{3}}
$$

où $k$ est la composante de Boltzmann, $T$ la température et $V$ le volume et où $d$ est la longueur de Debye définie par :

$$
d=\left(\frac{\varepsilon_{0} k T V}{e^{2} \sum_{i} z_{i}^{2} N_{i}}\right)^{1 / 2}
$$

où $z_{i}$ est le nombre de charge de l'espèce $i$ et $e$ la charge de l'électron.

L'expression finale de $G$ passe par la détermination des potentiels chimiques qui sont écrits à partir des fonctions de partition. Rappelons, à ce niveau, que les interactions de type coulombien assurent la convergence de la fonction de partition électronique en limitant son nombre de termes. Le dernier nombre quantique principal retenu $\left(n^{*}\right)$, cet état étant supposé hydrogénoide, est fixé différemment suivant les théories : des auteurs écrivent [23, 24]

$$
n^{*} \leqslant\left(\frac{Z_{\mathrm{eff}} \bar{d}_{+}}{a_{0}}\right)^{1 / 2}
$$

où $Z_{\text {eff }}$ est le nombre de charge effective et où $a_{0}$ et $\bar{d}_{+}$ sont respectivement le rayon de Bohr et la distance moyenne entre particules chargées :

$$
\bar{d}_{+}=\left(\frac{3}{4 \pi} \sum_{z \neq 0} n_{z}\right)^{1 / 3}
$$

où $n_{z}=N_{z} / V$ est la densité de particule de l'espèce chimique $z$, d'autres auteurs définissent $n^{\prime *}$ à partir de l'abaissement du potentiel d'ionisation $\Delta E_{i}[22,25]$

$$
n^{\prime *} \leqslant\left(\frac{z^{2} E_{\mathrm{H}}}{\Delta E_{i}}\right)^{1 / 2}
$$


où $E_{\mathrm{H}}$ est l'énergie d'ionisation de l'hydrogène et où $\Delta E_{i}$ s'écrit :

$$
\Delta E_{i}=\frac{(z+1) e^{2}}{4 \pi \varepsilon_{0} d}
$$

Il existe, entre ces deux nombres quantiques principaux, une relation d'inégalité : $n^{\prime *}>n^{*}$. Cependant on montre $[26,27]$ qu'à la pression atmosphérique les résultats obtenus pour les fonctions de partition (et leurs dérivées) avec les deux théories de limitation, divergent lorsque l'espèce est en voie de disparition c'est-à-dire que ces deux théories conduisent à la même composition d'équilibre sauf pour les espèces minoritaires qui alors n'ont plus d'influence sur les propriétés de transport.

Remarquons que la composition intervient, quelle que soit la théorie de limitation, dans le calcul des fonctions de partition par l'intermédiaire de $\bar{d}_{+}$ou de $d$. Finalement notons que ce champ de Coulomb amène aussi une modification de la pression et nous avons :

$$
P=\frac{k T}{V} \sum_{i} N_{i}-\frac{k T}{24 \pi d^{3}}
$$

1.2 Calcul Des PROPRiÉtés DE TRANSPORT. L'étude théorique cinétique classique des coefficients de transport d'un plasma se fait à l'aide de l'équation intégro-différentielle de Boltzmann résolue, par exemple, par la méthode de Chapman-Enskog [28]. Cette théorie, établie pour des gaz neutres sans énergie interne et non réactifs, doit être aménagée dans le cas des plasmas $[28,29]$ et les résultats pour être valables devront être basés sur une résolution à « un ordre plus élevé » de l'équation de Boltzmann et tenir compte des réactions intervenant dans le plasma (dissociation des molécules, ionisation) et de l'énergie ionisation et de l'énergie interne des particules. Nous avons utilisé la formulation simplifiée de Devoto [30] qui découple les propriétés de transport des électrons des autres espèces du fait que la masse de l'électron $\left(m_{1}\right)$ est très faible par rapport à celle des autres espèces $\left(m_{i}\right)$ et repris les notations de Gorse [18]. Notons que pour des raisons de commodités dans le formalisme, l'indice 1 est réservé aux électrons.

1.2.1 Intégrales de collision. - Les propriétés de transport sont gouvernées par les collisions interparticulaires qui sont présentées, dans le formalisme, au travers des intégrales de collision $[28,29]$ :

$$
\begin{aligned}
\bar{\Omega}^{(l, s)}=\frac{\bar{Q}^{(l, s)}}{\pi}=\frac{2(l+1)}{\pi(s+1) !\left[2 l+1-(-1)^{l}\right]} \times \\
\times \int_{0}^{\infty} \exp (-X) X^{s+1} Q^{l}(\varepsilon) \mathrm{d} X
\end{aligned}
$$

avec $X=\varepsilon / k T$, où $\varepsilon$ est l'énergie relative des particules et $Q^{l}(\varepsilon)$ est la section efficace de transfert de moment. Le niveau d'approximation retenu fixe le nombre de paires $(l, s)$ à considérer c'est-à-dire le nombre d'intégrales de collision à calculer [28, 29].

L'obtention de ces intégrales se fait alors par deux méthodes :

- soit la section efficace de transfert $Q^{l}$ est connue (expérimentalement ou théoriquement) et l'on intègre numériquement l'équation précédente, c'est en général le cas des collisions électron-neutre,

- soit le potentiel d'interaction lors d'une collision (relié directement à $Q^{l}$ ) est connu, la détermination de $\bar{\Omega}^{(l, s)}$ passe alors par des tables.

1.2.2 Coefficient de diffusion binaire. - Il correspond au coefficient de diffusion ordinaire de la théorie cinétique pour des mélanges comprenant deux espèces $i$ et $j$ et pour la première approximation. On montre que [31]:

$$
D_{i j}=\frac{3(2 k T)^{1 / 2}}{16 \sqrt{\pi} n \bar{\Omega}_{i j}^{(2,2)}}\left(\frac{M_{i}+M_{j}}{M_{i} M_{j}}\right)^{1 / 2}
$$

où $n$ est la densité totale $(n=N / V)$ et $M_{i}$ la masse molaire de l'espèce $i$.

Par passage à la limite on peut obtenir $D_{i i}$ bien que le coefficient de diffusion ordinaire lui soit nul.

1.2.3Viscosité. - La viscosité, indépendante des propriétés des électrons, peut être calculée avec une bonne approximation au moyen de la $1^{\text {re }}$ approximation de la méthode de Chapman-Enskog :

$\eta=\left|\begin{array}{cccc}H_{22} & \ldots & H_{2 N} & x_{2} \\ \vdots & & \vdots & \vdots \\ H_{N 2} & \ldots & H_{N N} & x_{N} \\ x_{2} & \ldots & x_{N} & 0\end{array}\right| \quad|\quad| \begin{array}{ccc}H_{22} & \ldots & H_{2 N} \\ \vdots & & \vdots \\ H_{N 2} & \ldots & H_{N N}\end{array} \mid$

où $x_{i}=N_{i} / N$ est la fraction molaire de l'espèce $i$.

Les autres coefficients sont donnés dans le tableau I [30].

1.2.4 Conductibilité thermique. - Elle est la somme de trois composantes : de translation, de réaction et interne

$$
\lambda_{\text {tot }}=\lambda_{\mathrm{tr}}+\lambda_{\mathrm{R}}+\lambda_{\mathbf{l}} .
$$

1 2.4.1 Conductibilité de translation. - Dans la formulation simplifiée de Devoto, on exprime la conductibilité de translation des particules lourdes à la deuxième approximation et celle des électrons à la troisième approximation.

La première $\left(\lambda_{\mathrm{tr}}^{\mathrm{h}}\right)$ s'écrit sous une forme identique à celle de la viscosité et les coefficients sont donnés dans le tableau I [30].

La deuxième, celle des électrons, dépend uniquement des collisions électron-électron et électronautres particules, elle s'écrit :

$$
\lambda_{\mathrm{tr}}^{\mathrm{e}}=\frac{75}{8} n_{1}^{2}\left(\frac{2 \pi k T}{m_{1}}\right)^{1 / 2} \frac{q^{(2,2)}}{q^{(1,1)} q^{(2,2)}-\left(q^{(1,2)}\right)^{2}} .
$$


Tableau I. - Equations utilisées.

[Working equations.]

\begin{tabular}{|c|c|c|}
\hline Grandeurs & \multicolumn{2}{|l|}{ Formalisme } \\
\hline$\eta$ & $\begin{array}{l}H_{i i}=\frac{x_{i}^{2}}{\eta_{i i}}+\sum_{\substack{k \neq i \\
k=2}}^{N} \frac{2 N_{\mathrm{av}} x_{i} x_{k}}{n D_{i k}\left(M_{i}+M_{k}\right)}\left(1+\frac{3}{5} \frac{M_{k}}{M_{i}} A_{i k}^{*}\right) \\
H_{i j}=-\frac{2 N_{\mathrm{av}} x_{i} x_{j}}{n D_{i j}\left(M_{i}+M_{j}\right)}\left(1-\frac{3}{5} A_{i j}^{*}\right)\end{array}$ & $\begin{aligned} \eta_{i i} & =\frac{5}{16 \sqrt{\pi}} \frac{\left(M_{i} R T\right)^{1 / 2}}{N_{\mathrm{av}} \bar{\Omega}_{i i}^{(2,2)}} \\
A_{i j}^{*} & =\frac{\bar{\Omega}_{i j}^{(2,2)}}{\bar{\Omega}_{i j}^{(1), 1)}}\end{aligned}$ \\
\hline$\lambda_{\mathrm{tr}}^{\mathrm{h}}$ & $\begin{array}{l}L_{i i}=-\frac{4 x_{i}^{2}}{\lambda_{i i}}-\sum_{\substack{k \neq i \\
k=2}}^{N} \frac{16 N_{\mathrm{av}} x_{i} x_{k}}{25 n R D_{i k}\left(M_{i}+M_{k}\right)^{2}}\left(\frac{15}{2} M_{i}^{2}+\frac{25}{4} M_{k}^{2}-3 M_{k}^{2} B_{i k}^{*}+4 M_{i} M_{k} A_{i k}^{*}\right) \\
L_{i j}=\frac{16 N_{\mathrm{av}} x_{i} x_{j} M_{i} M_{j}}{25 n R D_{i j}\left(M_{i}+M_{j}\right)^{2}}\left(\frac{55}{4}-3 B_{i j}^{*}-4 A_{i j}^{*}\right)\end{array}$ & $\begin{array}{l}\lambda_{i i}=\frac{75}{64 \sqrt{\pi}} \frac{R^{3 / 2}}{N_{\mathrm{av}} \bar{\Omega}_{i i}^{(2,2)}}\left(\frac{T}{M_{i}}\right)^{1 / 2} \\
B_{i j}^{*}=\frac{5 \bar{\Omega}_{i j}^{(1,2)}-4 \bar{\Omega}_{i j}^{(1,3)}}{\bar{\Omega}_{i j}^{(1,1)}}\end{array}$ \\
\hline$\lambda_{R}$ & $A_{i j}=A_{j i}=\sum_{k=1}^{N-1} \sum_{l=k+1}^{N} \frac{N_{\mathrm{av}} x_{k} x_{l}}{n D_{k l}}\left(\frac{a_{i k}}{x_{k}}-\frac{a_{i l}}{x_{l}}\right)\left(\frac{a_{i k}}{x_{k}}-\frac{a_{j l}}{x_{l}}\right)$ & \\
\hline$\lambda_{\mathrm{tr}}^{\mathrm{e}}$ & $\begin{array}{l}q^{(0,0)}=8 n_{1} \sum_{i=2}^{N} n_{i} \bar{Q}_{1 i}^{(1,1)} \\
q^{(0,1)}=8 n_{1} \sum_{i=2}^{N} n_{i}\left(\frac{5}{2} \bar{Q}_{1 i}^{(1,1)}-3 \bar{Q}_{1 i}^{(1,2)}\right) \\
q^{(0,2)}=8 n_{1} \sum_{i=2}^{N} n_{i}\left(\frac{35}{8} \bar{Q}_{1 i}^{(1,1)}-\frac{21}{2} \bar{Q}_{1 i}^{(1,2)}+6 \bar{Q}_{1 i}^{(1,3)}\right) \\
q^{(1,1)}=8 \sqrt{2} n_{1}^{2} Q_{11}^{(2,2)}+8 n_{1} \sum_{i=2}^{N} n_{i}\left(\frac{25}{4} \bar{Q}_{1 i}^{(1,1)}-15 \bar{Q}_{1 i}^{(1,2)}+12 \bar{Q}_{1 i}^{(1,3)}\right) \\
q^{(1,2)}=8 \sqrt{2} n_{1}^{2}\left(\frac{7}{4} \bar{Q}_{1 i}^{(2,2)}-2 \bar{Q}_{1 i}^{(2,3)}\right)+8 n_{1} \sum_{i=2}^{N} n_{i}\left(\frac{175}{16} \bar{Q}_{1 i}^{(1,1)}-\frac{315}{8} \bar{Q}_{1 i}^{(1,2)}+57 \bar{Q}_{1 i}^{(1,3)}\right. \\
q^{(2,2)}=8 \sqrt{2} n_{1}^{2}\left(\frac{77}{18} Q_{1 i}^{(2,2)}-7 Q_{1 i}^{(2,3)}+5 Q_{1 i}^{(2,4)}\right)+8 n_{1} \sum_{i=2}^{N} n_{i}\left(\frac{1225}{2} \bar{Q}_{1 i}^{(1,1)}-\frac{735}{8} \bar{Q}_{1 i}^{(1,2)}\right.\end{array}$ & $\begin{array}{l}0 \\
\left.0 \bar{Q}_{1 i}^{(1,4)}\right) \\
\frac{399}{2} \bar{Q}_{1 i}^{(1,3)}-210 \bar{Q}_{1 i}^{(1,4)}+ \\
\left..+90 \bar{Q}_{1 i}^{(1,5)}\right)\end{array}$ \\
\hline
\end{tabular}

Les expressions de $q$ sont données dans le tableau I $[29,30]$.

1.2.4.2 Conductibilité de réaction et interne.

Nous regroupons ces deux composantes de la conductibilité car elles ne peuvent être développées qu’à la première approximation [28]. En effet pour l'établissement des relations on utilise le fait que le coefficient de diffusion binaire est symétrique $\left(D_{i j}=D_{j i}\right)$ mais cette propriété ne semble pas se prolonger audelà de cet ordre.

La conductibilité interne du plasma est liée à la présence de degrés internes de libertés des particules, à partir d'Eucken, on écrit [28] :

$$
\lambda_{\mathrm{I}}=\sum_{i=2}^{N}\left(\lambda_{\mathrm{I}}\right)_{i}\left(\sum_{j=2}^{N} \frac{x_{j}}{x_{i}} \frac{D_{i i}}{D_{i j}}\right)^{-1},
$$

où $\left(\lambda_{\mathrm{I}}\right)_{i}$ est la conductibilité thermique interne de la $i$-ième espèce définie telle que :

$$
\left(\lambda_{\mathrm{I}}\right)_{i}=\frac{n D_{i i}}{N_{\mathrm{av}}}\left(C_{\mathrm{p}}(i)-\frac{5}{2} R\right),
$$

où $C_{\mathrm{p}}(i)$ est la chaleur spécifique de la $i$-ì̀me espèce.

La conductibilité de réaction peut être calculée au moyen de la théorie de Butler et Brokaw [28]. Les $\mu$ réactions s'écrivent comme suit :

$$
\sum_{k=1}^{N} a_{i k} B^{k}=0 \quad i \in[1, \mu],
$$

où $B^{k}$ représente le $k$-ième espèce chimique.

La chaleur de réaction se détermine en fonction des enthalpies molaires $H_{k}$ de chaque espèce :

$$
\Delta H_{i}=\sum_{k=1}^{N} a_{i k} H_{k} \quad i \in[1, \mu] .
$$

La conductibilité thermique de réaction $\left(\lambda_{R}\right)$ s'exprime alors comme la viscosité, les enthalpies de réac- 
tion remplaçant les fractions molaires dans le formalisme.
1.2.5 Conductivité électrique. - Pour la troisième approximation, elle s'écrit :

avec :

$$
\sigma=\frac{3}{2} e^{2} n_{1}^{2}\left(\frac{2 \pi}{m, k T}\right)^{1 / 2}\left(q^{(1,2)} q^{(2,2)}-\left(q^{(1,2)}\right)^{2}\right) / D
$$

$$
D=q^{(0,0)}\left(q^{(1,1)} q^{(2,2)}-\left(q^{(1,2)}\right)^{2}\right)+q^{(0,1)}\left(q^{(1,2)} q^{(0,2)}-q^{(0,1)} q^{(2,2)}\right)+q^{(0,2)}\left(q^{(0,1)} q^{(1,2)}-q^{(0,2)} q^{(1,1)}\right)
$$

Les expressions de $q$ sont données dans le tableau I [30].

1.3 Potentiels D'inTeraction ET INTÉGRALES DE COLLISION. - Les potentiels d'interaction interparticulaires, nécessaires à la détermination des intégrales de collision, sont obtenus théoriquement ou expérimentalement de manière de plus en plus précise et dans le même temps leurs formes analytiques sont devenues plus complexes. Nous allons donc voir, successivement, les collisions chargée-chargée, neutreneutre et neutre-chargée en examinant quelles formes analytiques du potentiel peuvent décrire les interactions et comment on peut en déduire les intégrales de collision.

1.3.1 Collision chargée-chargée. - Pour un potentiel de type Coulombien classique $\left(V=z_{i} z_{j} e^{2} / 4 \pi \varepsilon_{0} r\right)$ les intégrales de collisions sont divergentes. Pour les rendre finies on introduit un paramètre de coupure, distance au-dessus de laquelle les interactions interparticulaires n'ont plus lieu et l'on écrit le potentiel de Coulomb avec écran sous la forme suivante :

$$
V=z_{i} z_{j} e^{2} \exp (-r / d) / 4 \pi \varepsilon_{0} r,
$$

où $\varepsilon_{0}$ est la permittivité du vide.

Les intégrales de collision correspondantes sont calculées par Liboff [32] qui ne différencie pas les potentiels attractifs des répulsifs. C'est pourquoi il est préférable d'utiliser les tables de Mason et al. [33] complétées par Devoto [34] pour atteindre la troisième approximation pour les coefficients de transport des électrons.

Les interactions étant brèves (à l'intérieur de la sphère de Debye) on admet pour le calcul des intégrales de collision, la quasi-immobilité ionique et il est préférable de prendre en compte uniquement la contribution électronique, soit :

$$
d_{\mathrm{e}}=\left(\frac{\varepsilon_{0} k T V}{e^{2} N_{\mathrm{e}}}\right)^{1 / 2} .
$$

Cela a pour effet d'augmenter la valeur des intégrales qui seront calculées pour chaque composition dont elles dépendent à travers $d_{\mathrm{e}}$.

1.3.2 Collision neutre-neutre. - Un très grand nombre de formes analytiques de potentiel peuvent représenter ce type d'interaction, nous les avons séparées en deux catégories : celles pour lesquelles il existe des tables d'intégrales de collision et les autres.
1.3.2.1 Forme du potentiel et tables d'intégrales. a) Formes analytiques à un seul terme. - Ce sont les deux formes les plus simples :

- Les potentiels puissances, en $V= \pm V_{0} r^{-n}$, qui sont utilisés aussi bien dans la zone attractive (de $n=3$ pour une interaction molécule dipolaire-molécule dipolaire à $n=10$ pour une collision quadripole induit-quadripole induit) que dans la zone répulsive. Les intégrales de collision correspondantes sont obtenues à l'aide des tables de Kihara et al. [35], comme suit :

$$
\begin{aligned}
\bar{\Omega}^{(l, s)}=\frac{4(l+1)}{(s+1) !\left(2 l+1-(-1)^{l}\right)} \times \\
\quad \times\left(\frac{n V_{0}}{k T}\right)^{2} / n \Gamma\left(s+2-\frac{2}{n}\right) A^{(l)}(n),
\end{aligned}
$$

où les $A^{(l)}(n)$ sont les grandeurs tabulées et $\Gamma$ est la fonction factorielle.

- La forme exponentielle, $V= \pm V_{0} \exp (-B r)$, apparaît, en général :

- pour des ajustements dans la zone répulsive $(V>0)$, dans ce cas, Monchick [36] obtient (en posant $\left.\alpha_{\mathrm{m}}=\log \left(V_{0} / k T\right)\right)$ les intégrales sous la forme suivante :

$\bar{\Omega}^{(l, s)}=\frac{2(l+1)}{(s-1) !\left(2 l+1-(-1)^{l}\right)} 8\left(\frac{\alpha_{\mathrm{m}}}{B}\right)^{2} I(l, s)$,

où les $I(l, s)$ sont tabulées;

- pour un potentiel attractif, Brokaw [37] fourni la possibilité de calculer les intégrales en utilisant les deux résultats précédents.

B) Formes analytiques à plusieurs termes. - Pour une collision pouvant conduire à un état lié, l'existence d'un puits de potentiel fait que si l'on veut tenir compte du potentiel dans son ensemble, la forme analytique doit avoir au minimum deux termes pour représenter la zone d'attraction à longue distance (potentiel négatif) et la zone violemment répulsive à courte distance (potentiel positif). Pour ces formes analytiques nous avons retenu :

- Le potentiel de Morse [38] :

$V(r)=D_{\mathrm{e}}\left[\exp \left(-2 \beta\left(r-r_{\mathrm{m}}\right)\right)-2 \exp \left(-\beta\left(r-r_{\mathrm{m}}\right)\right)\right]$,

où $D_{\mathrm{e}}$ est la profondeur du puits de potentiel et $r_{\mathrm{m}}$ la distance interatomique correspondant à l'équilibre 
(c'est-à-dire au minimum de $V(r)$ ). Ce potentiel s'annule pour $r_{0}$ défini comme suit :

$$
r_{0}=r_{\mathrm{m}}-\beta^{-1} \log 2 .
$$

Pour l'obtention des intégrales de collision, deux possibilités de tables : d'une part celles de Samoilov et al. $[39,40]$ qui prend un potentiel de Morse corrigé pour qu'il devienne infini lorsque $r<0,3 r_{0}$, d'autre part celles de Smith et al. [41] qui le conserve sous sa forme classique.

- Le potentiel $n-6$ de Lennard-Jones [42] aussi appelé potentiel de Mie quand il est écrit sous la forme suivante :

$$
V=\frac{6 D_{\mathrm{e}}}{n-6}\left(\left(\frac{r_{\mathrm{m}}}{r}\right)^{n}-\frac{n}{6}\left(\frac{r_{\mathrm{m}}}{r}\right)^{6}\right)
$$

avec

$$
r_{0}=\left(\frac{n}{6}\right)^{-\frac{1}{n-6}} \times r_{\mathrm{m}} .
$$

La forme la plus utilisée est le potentiel 12-6 mais certains chercheurs préfèrent le potentiel 9-6 [43, 44]. Pour les intégrales de collision on dispose des tables très complètes de Klein et al. [45], cependant dans le cas du potentiel 12-6 on utilise la formule empirique de Neufeld et al. [46] dont nous conservons les notations :

$$
\begin{aligned}
\bar{\Omega}^{(l, s)}=r_{0}^{2}\left(\frac{A}{T^{*} B}+\frac{C}{\exp \left(D T^{*}\right)}+\frac{E}{\exp \left(F T^{*}\right)}+\right. \\
\left.\quad+\frac{G}{\exp \left(H T^{*}\right)}+R T^{* B} \sin \left(S T^{* W}-P\right)\right)
\end{aligned}
$$

où $T^{*}$ est une température réduite telle que $T^{*}=k T / D_{\mathrm{e}}$.

- Le potentiel de Buckingham s'écrit $[47,48]$ :

$$
V=\frac{D_{\mathrm{e}}}{\alpha-6}\left[6 \exp \left(\alpha\left(1-\frac{r}{r_{\mathrm{m}}}\right)\right)-\alpha\left(\frac{r_{\mathrm{m}}}{r}\right)^{6}\right] \text {. }
$$

Pour cette forme Mason [48] a tabulé une grandeur $Z^{(l, s)}$ à partir de laquelle on dérive $\bar{\Omega}^{(l, s)}$ par la relation :

$$
\bar{\Omega}^{(l, s)}=r_{\mathrm{m}}^{2}\left(T^{*}(1-6 / \alpha)\right)^{-1 / 3} Z^{(l, s)} .
$$

Finalement nous citons le potentiel de Stockmeyer qui est la somme du potentiel 12-6 de Lennard-Jones et du potentiel existant entre deux molécules ayant un moment dipolaire (en $1 / r^{3}$ ) car Monckick et al. [49] fournissent les intégrales correspondantes.

1.3.2.2 Autres formes de potentiel. - Dans la littérature les formes analytiques du potentiel deviennent de plus en plus complexes afin d'améliorer les ajustements avec les résultats expérimentaux ou théoriques, mais elles ne permettent pas un calcul direct des intégrales de collision. Nous rapportons certaines formes que nous avons trouvées lors de notre recherche et pour alléger le formalisme nous introduisons deux grandeurs réduites définies par:

$$
x_{\mathrm{m}}=r / r_{\mathrm{m}} ; \quad C_{2 i+4}=\frac{c_{2 i+4}}{D_{\mathrm{e}} r_{\mathrm{m}}^{2 i+4}} .
$$

Dans le tableau II sont données des formes dérivées des potentiels de Lennard-Jones et de Buckingham.

Dans le tableau III, sont signalés certains potentiels à formes analytiques variables qui découplent les régions à forte et faible interactions par un découpage : à chaque plage correspond une forme différente avec des conditions de continuité du potentiel et de sa dérivée aux points de jonction.

1.3.3 Collision neutre-charge. - Les intégrales de collision s'obtiennent comme dans le cas des interactions neutre-neutre, à l'exception de celles de type diffusion $(l=1)$ lors de chocs $\mathbf{M}-\mathrm{M}^{+}$(transfert résonnant) [50]. Il faut alors connaître la section efficace de transfert de charge $Q_{\mathrm{tr}}^{(n)}$ (l'indice $n$ pour la $n$ ième courbe de potentiel) que l'on écrit sous la forme suivante [51] :

$$
Q_{\mathrm{tr}}^{(n)}=\frac{1}{2}\left(A_{n}-B_{n} \log \left(g^{\prime}\right)\right)^{2}
$$

où $A_{n}$ et $B_{n}$ sont des coefficients obtenus expérimentalement ou théoriquement (exprimés en $\AA$ ) et où $g^{\prime}$ est la vitesse relative (exprimée en $\mathrm{cm} / \mathrm{s}$ ). Les intégrales de collision sont alors calculées par [52] :

$$
\begin{aligned}
\bar{\Omega}_{n}^{(l, s)}=\frac{1}{\pi}\left\{A_{n}-A_{n} B_{n} x-\left(\frac{B_{n} x}{2}\right)^{2}+\frac{B_{n} \xi}{2}\left(B_{n} x\right.\right. & \left.-2 A_{n}\right)+\frac{B_{n}^{2}}{4}\left(\frac{\pi^{2}}{6}-\sum_{n=1}^{s+1} \frac{1}{n^{2}}+\xi\right)+ \\
& \left.+\frac{B_{n}}{2}\left(B_{n}(x+\xi)-2 A_{n}\right) \log \left(\frac{T}{M}\right)+\left(\frac{B_{n}}{2} \log \left(\frac{T}{M}\right)\right)^{2}\right\},
\end{aligned}
$$

où $x=\log (4 R), R$ étant la constante des gaz parfaits (exprimée en C.G.S.) où $\xi=\sum_{n=1}^{s+1} \frac{1}{n}-\bar{\gamma}, \bar{\gamma}=0,577216$ étant la constante d'Euler et où $M$ est la masse molaire.

Pour une détermination théorique de $A_{n}$ et $B_{n}$, on effectue la différence de potentiel pair-impair $(\mathrm{g}-\mathrm{u})$ pour chaque couple de potentiel et l'on effectue un ajustement tel que [52] :

$$
\left|V_{\mathrm{g}}^{(n)}-V_{\mathrm{u}}^{(n)}\right|=V_{0}^{(n)} \exp \left(-\alpha^{(n)} r\right) .
$$

De $V_{0}^{(n)}$ et $\alpha^{(n)}$ ainsi obtenus et par résolution de l'équation transcendante en $b_{\mathrm{e}}^{(n)}$ et $g^{\prime}$ [53] :

$$
b_{\mathrm{e}}^{(n)} \exp \left(-2 \alpha^{(n)} b_{\mathrm{e}}^{(n)}\right)=\frac{\pi}{8} \frac{\alpha^{(n)} \hbar^{2} g^{\prime 2}}{V_{0}^{(n) 2}},
$$


Tableau II. - Formes analytiques de potentiel d'interaction.

[Analytic forms for interaction potentials.]

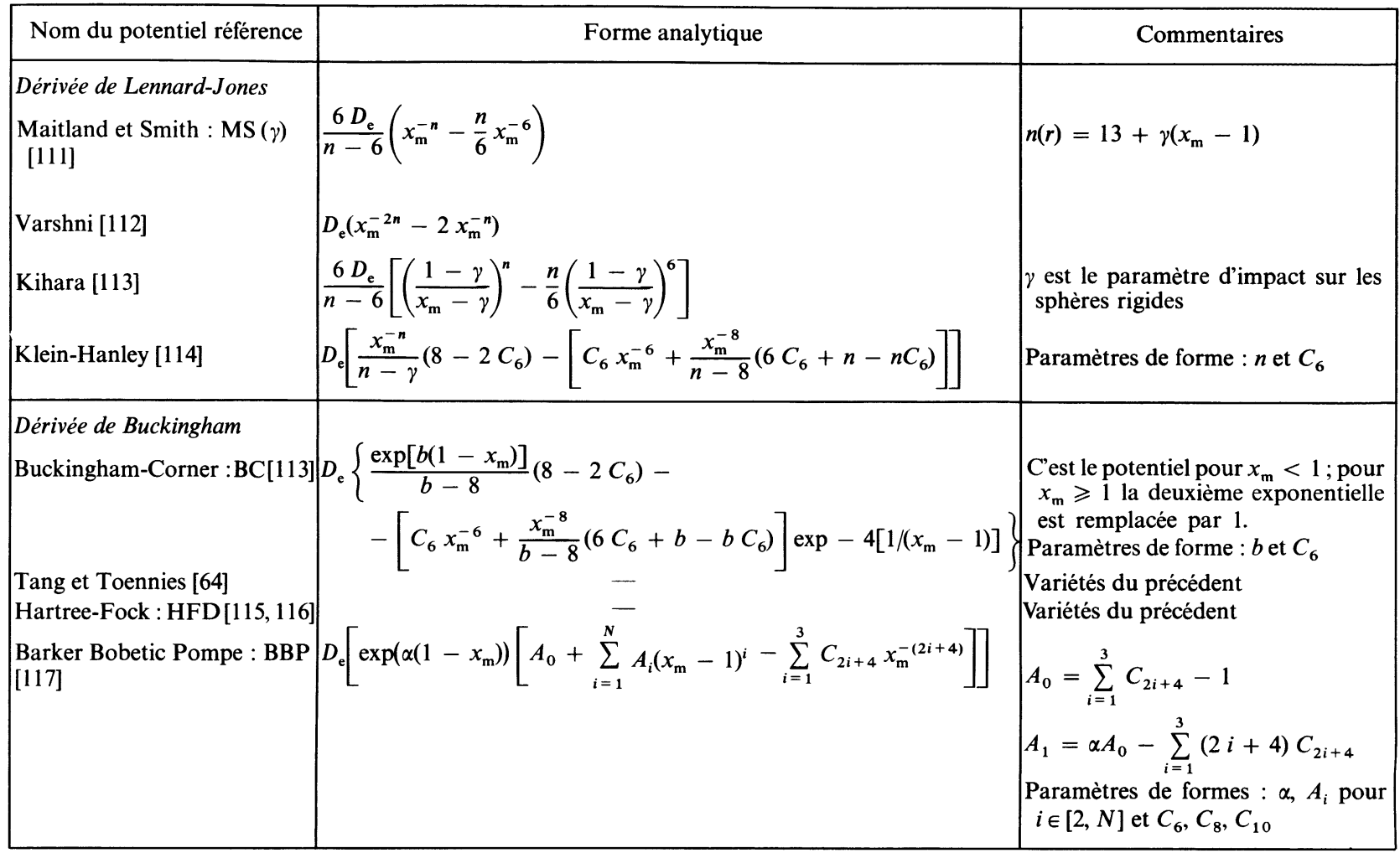

Tableau III. - Formes analytiques de potentiel d'interaction.

[Analytic forms for interaction potentials.]

\begin{tabular}{|c|c|c|}
\hline Nom du potentiel référence & Forme analytique & Commentaires \\
\hline Double Lennard-Jones [118] & $\begin{array}{l}\cdot \frac{m D_{\mathrm{e}}}{n_{1}-m}\left(x_{\mathrm{m}}^{-n_{1}}-\frac{n_{1}}{m} x_{\mathrm{m}}^{-m}\right) ; \quad x_{\mathrm{m}}<1 \\
-\frac{6 D_{\mathrm{e}}}{n_{2}-6}\left(x_{\mathrm{m}}^{-n_{2}}-\frac{n_{2}}{6} x_{\mathrm{m}}^{-6}\right) ; \quad x_{\mathrm{m}} \geqslant 1\end{array}$ & Paramètres de forme : $n_{1}, m, n_{2}$ \\
\hline $\begin{array}{l}\text { Morse-Spline-Van der Waals } \\
\text { MSV [119] }\end{array}$ & 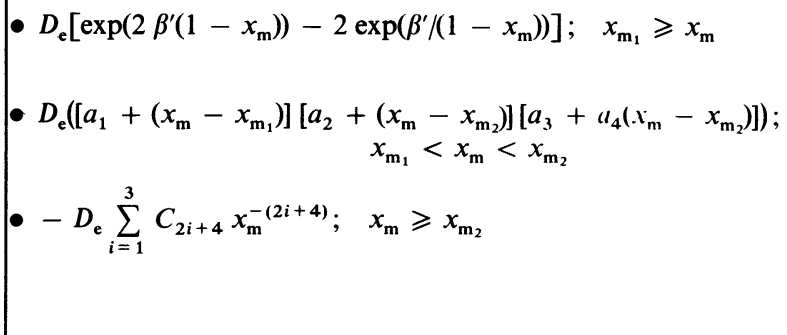 & $\begin{array}{l}\text { - } a_{1}, a_{2}, a_{3}, a_{4} \text { sont fixées par les condi- } \\
\text { tions de continuité en } x_{\mathrm{m}} \\
-r_{1}=r_{\mathrm{m}}\left(1-\beta^{\prime-1} \log 2\right) \text { et } r_{2}=1,6 r_{\mathrm{m}} \\
\text { (en général) } \\
\text { Paramètres de forme : } \beta^{\prime}, C_{6}, C_{8}, C_{10} . \\
\begin{array}{l}\text { Formé de la même manière (E pour } \\
\text { Exponentiel) }\end{array}\end{array}$ \\
\hline SPF Duham : SPFD $[121,122]$ & $\begin{array}{l}\text { - } D_{\mathrm{e}}\left[b_{0}\left(1-\frac{1}{x_{\mathrm{m}}}\right)^{2}\left[1+\sum_{i=1}^{N} b_{i}\left(1-\frac{1}{x_{\mathrm{m}}}\right)^{i}\right]-1\right] ; x<x_{\mathrm{f}} \\
\text { - } D_{\mathrm{e}} \sum_{i=1}^{3} C_{2 i+4} x_{\mathrm{m}}^{-(2 i+4)} ; \quad x \geqslant x_{\mathrm{f}}\end{array}$ & $\begin{array}{l}\text { - En général } x_{\mathrm{f}}>x_{\mathrm{m}} \\
\text { - La continuité en } x_{\mathrm{f}} \text { fixe } b_{N} \text { et } b_{N-1} \\
\text { - Paramètres de forme : } b_{1} \ldots, b_{N-2} \text { et } \\
C_{6}, C_{8}, C_{10} \\
\text { - Peut osciller pour } x_{\mathrm{m}} \leqslant 0,65\end{array}$ \\
\hline
\end{tabular}


on déduit [53] :

$$
Q_{\mathrm{tr}}^{(n)}=\frac{1}{2} \pi b_{\mathrm{e}}^{(n) 2}+\frac{\pi^{3}}{16 \alpha^{(n)}}\left(\alpha^{(n)} b_{\mathrm{e}}^{(n)}+1+\frac{1}{2 \alpha^{(n)} b_{\mathrm{e}}^{(n)}}\right) .
$$

Après ajustement sur les couples $\left(Q_{\mathrm{tr}}^{(n)}, \log \left(g^{\prime}\right)\right)$ obtenus un à un, on obtient $A_{n}$ et $B_{n}$.

On montre [54] qu'écrire

$$
Q_{\mathrm{tr}}^{(n)}=\frac{1}{2} \pi b_{\mathrm{e}}^{(n) 2},
$$

et résoudre

$$
b_{\mathrm{e}}^{(n)} \exp \left(-2 \alpha^{(n)} b_{\mathrm{e}}^{(n)}\right)=\frac{2}{\pi^{3}} \frac{\alpha^{(n)} \hbar^{2} g^{\prime 2}}{V_{0}^{(n) 2}},
$$

est une bonne approximation et ce sont ces deux dernières relations que nous avons utilisées.

1.3.4 Conclusion. - Il faut remarquer que si les formes analytiques du potentiel ont beaucoup évolué récemment, les tables permettant la détermination des intégrales de collision n'existent que pour les formes simples connues lors de leurs établissements, à l'exception de celles de Van Brunt et al. [55] mais qui sont inutilisables car trop incomplètes. C'est pourquoi, en partant des formes potentielles plus complexes de la littérature on est amené à faire les ajustements, à l'aide des potentiels pour lesquels on dispose des tables, dans les domaines d'énergies ayant de l'importance pour la détermination des propriétés de transport. Ces plages d'énergies sont fixées par les règles d'Hirchfelder-Eliason [56] :

— états répulsifs $\ldots \ldots \ldots \ldots \ldots \ldots \ldots \ldots \ldots \ldots \ldots \ldots \ldots$
— états liés $\ldots \ldots \ldots \ldots \ldots \ldots$

Finalement les collisions entre deux espèces peuvent se faire suivant plusieurs courbes de potentiels, dans ce cas on utilise pour le calcul des coefficients de transport une intégrale de collision moyenne définie ainsi [18] :

$$
\left\langle\bar{\Omega}^{(l, s)}\right\rangle=\frac{\sum_{n} \rho_{n} \bar{\Omega}_{n}^{(l, s)}}{\sum_{n} \rho_{n}},
$$

où $\rho_{n}$ est le poids statistique du même état.

2. Application au mélange $\mathbf{A r}-\mathbf{H}_{2}$ - Pour des pressions voisines de la pression atmosphérique et pour une température maximale de $15000 \mathrm{~K}$, nous ne tenons compte que des espèces suivantes : $\mathrm{Ar}, \mathrm{Ar}^{+}, \mathrm{H}_{2}, \mathrm{H}$, $\mathrm{H}^{+}$et e.

Dans un premier temps nous étudions les collisions une à une et pour des raisons de facilité de calcul, plutôt que d'introduire des tableaux (pour les fonctions de partition et de leurs dérivées ainsi que pour les intégrales de collision) nous utilisons des polynômes d'ajustement définis ainsi :

$$
\bar{\Omega}^{(l, s)}=a(1) \log (T)+\sum_{i=1}^{8} a(i) T^{n(i)} .
$$

Nous avons limité le nombre de coefficients $a(i)$ à huit mais les exposants $n(i)$ de $T$ varient entre -9 et +9 et sont aussi stockés sur fichier.

Puis nous commentons nos résultats : composition du plasma et propriétés de transport.

2.1 INTÉGRALES DE COLLISION. - L'ajustement se fait sur 146 points de $500 \mathrm{~K}$ à $15000 \mathrm{~K}$, par pas de $100 \mathrm{~K}$ et les coefficients sont rapportés dans le tableau IV.

2.1.1 Interaction Ar-Ar. - Pour cette interaction qui est particulièrement étudiée nous avons retenu le potentiel MSV de Parson et al. [57] bien qu'il ne soit pas assez répulsif aux basses énergies. Cependant les intégrales de collision calculées par ce potentiel ne sont inférieures que de $5 \%$ à $500 \mathrm{~K}$ par rapport à celles obtenues avec le potentiel 12-6 de Clifford et al. [58] déterminé à partir de données sur la viscosité à basse température (jusqu'à $500 \mathrm{~K}$ ). De plus, à plus hautes énergies il est en bon accord avec les résultats de Rol [59] qui utilise les données expérimentales de Amdur et al. [60]. Pour le calcul des intégrales nous n'avons conservé que le potentiel de Morse, négligeant un bon ajustement de la partie dispersive, ce qui est raisonnable compte tenu de la faible hauteur du puits. Ces coefficients sont :

$$
D_{\mathrm{e}}=0,01208 \mathrm{eV} ; \quad r_{\mathrm{m}}=3,760 \AA ; \quad \beta r_{\mathrm{m}}=6,279 .
$$

Les intégrales de collision (les coefficients du polynôme sont donnés dans le tableau IV), obtenues avec les tables de Smith et al. [41], sont toujours supérieures à celles de Gorse [18] mais inférieures à celles que nous avons calculées à partir des potentiels plus récents (forme HFD) de Alhrichs et al. [61] et Aziz et al. [59].

2.1.2 Interaction Ar-H. - Des études, tant expérimentales [62, 63] que théoriques [64-66], ont été faites pour déterminer les valeurs de $D_{\mathrm{e}}$ et $r_{\mathrm{m}}$ de cette collision. Nous avons retenu le potentiel théorique de Brual et al. [66] qui est en bon accord avec les résultats expérimentaux de Bassi et al. [63] et que nous avons écrit sous la forme exponentielle répulsive avec :

$$
V_{0}=138,3 \mathrm{eV} ; \quad B=2,783 \AA^{-1} .
$$

2.1.3 Interaction Ar- $\mathrm{H}_{2}$. - Dunker et al. [67] écrit cette énergie potentielle sous la forme suivante :

$$
V(r, \theta)=V_{0}(r)+V_{2}(r) P_{2}(\cos \theta),
$$

où $P_{2}(\cos \theta)$ est le polynôme de Lagrange, où $V_{0}$ et $V_{2}$ sont deux potentiels MSV, dont le deuxième est légèrement modifié par l'introduction de coefficients d'anisotropie. Cette collision est importante aux basses énergies et alors la partie anisotrope est seulement une petite correction, nous avons donc intégré sur $\theta$ pour obtenir un potentiel sphérique de type Morse qui assure le meilleur ajustement avec les trois potentiels de Dunker et al. [67] (pour trois états de 
Tableau IV. - Coefficients du polynôme d'ajustement des intégrales de collision. Notation : $\oplus: 2,223958(4)$ $[-1]=2,223958 \times 10^{4} \times T^{-1}$.

[Coefficients of the fitting polynomes for the collision integrales. Notation : $\oplus$ :

$$
\left.2.223958(4)[-1]=2.223958 \times 10^{4} \times T^{-1} \text {. }\right]
$$

\begin{tabular}{|c|c|c|c|c|c|c|c|c|c|c|c|c|c|c|}
\hline Irseract: & eff $1=$ ients & $a_{1}$ & $a_{2}$ & & $a_{3}$ & & $a_{4}$ & & $a_{5}$ & & $a_{6}$ & & $a_{7}$ & $d_{3}$ \\
\hline $\bar{\Omega}(1,1)$ & $A r-A r$ & 2.920765 & $-21.44 y 71$ & c. & $-=.321156(-4)$ & 1 & $2.223958(4)^{\circ}$ & & $-2.130965(7)$ & $|-2|$ & $1.087277(10)$ & -3 & $-2.058106(12)-4$ & - \\
\hline $\bar{s}(2,2)$ & $\mathrm{Ar}-\mathrm{Ar}$ & 5.073893 & -39.98913 & $=$ & $-3.656305(-4)$ & 1 & $3.360280(4)$ & & $-3.240325(7)$ & -2 & $1.646927(10)$ & -3 & $-3.105186(12)-4$ & - \\
\hline $\bar{\Omega}(1,1)$ & $\mathrm{Ar}-\mathrm{H}$ & -1.174259 & 13.21492 & 0 & $1.495 E 14(-5)$ & 1 & $1.371302(3)$ & & $-7.275459(5)$ & -2 & $2.790412(8)$ & -3 & $-4.565439(10)-4$ & - \\
\hline $\bar{\Omega}^{(2,2)}$ & $\mathrm{Ar}-\mathrm{H}$ & -1.407138 & 16.09753 & 0 & $1.575 c 92(-5)$ & 1 & $1.404846(3)$ & & $-7.470748(5)$ & -2 & $2.866962(8)$ & -3 & $-4.690899(14)-4$ & - \\
\hline $\bar{\Omega}(1,1)$ & $\mathrm{Ar}-\mathrm{H}_{2}$ & - & 9.273573 & 0 & $-2.482854(-3)$ & 1 & $7.581996(-7)$ & 2 & $-1.296114(-10)$ & 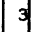 & $1.191796(-14)$ & 4 & $-5.547412(-19) 5$ & $1.026968(-23)$ \\
\hline $\bar{\Omega}(2,2)$ & $\mathrm{Ar}-\mathrm{H}_{2}$ & - & 10.24014 & 0 & $-2.485273(-3)$ & 1 & $7.519971(-7)$ & 2 & $|-1.278324(-10)|$ & & $1.166470(-14)$ & 4 & $-5.381062(-19) 5$ & $9.869505(-24) 0$ \\
\hline $\bar{\Omega}^{(1,1)}$ & $\mathrm{H}-\mathrm{H}$ & -1.161789 & 12.80087 & 0 & $1.893523(-5)$ & 1 & $1.365861(3)$ & -1 & $-6.701277(5)$ & -2 & $2.440833(8)$ & -3 & $-3.862216(10)-4$ & - \\
\hline $\bar{\Omega}(2,2)$ & $H-H$ & -1.067189 & 12.50676 & 0 & $-6.554111(-6)$ & 1 & $2.516254(3)$ & - & $-1.552865(6)$ & -2 & $6.314418(8)$ & -3 & $-1.059350(11)-4$ & - \\
\hline $\bar{\Omega}^{(1,1)}$ & $\mathrm{H}-\mathrm{H}_{2}$ & 0.7776049 & 8.371280 & 0 & $1.224154(-5)$ & 1 & $1.130945(3)$ & -1 & $-6.006357(5)$ & -2 & $2.305362(8)$ & -3 & $-3.774161(10)$ & - \\
\hline $\bar{\Omega}(2,2)$ & $\mathrm{H}-\mathrm{H}_{2}$ & -0.9625089 & 10.51257 & 0 & $1.319634(-5)$ & 1 & $1.167767(3)$ & -1 & $-6.225991(5)$ & -2 & $2.392675(8)$ & -3 & $-3.918410(10)-4$ & - \\
\hline $\bar{\Omega}(1,1)$ & $\mathrm{H}_{2}-\mathrm{H}_{2}$ & -0.9263416 & 10.72103 & 0 & $1.041954(-5)$ & 1 & $9.603987(2)$ & -1 & $-5.102140(5)$ & -2 & $1.958345(8)$ & -3 & $-3.205997(10)-4$ & - \\
\hline $\bar{\Omega}^{(2,2)}$ & $\mathrm{H}_{2}-\mathrm{H}_{2}$ & -1.092190 & 12.86188 & 0 & $1.087499(-5)$ & 1 & $9.796889(2)$ & -1 & $-5.212774(5)$ & -2 & $2.001334(8)$ & -3 & $-3.275572(10)$ & - \\
\hline $\bar{\Omega}(1,1)$ & $\mathrm{Ar}_{-} \mathrm{Ar}^{+}$ & -2.850735 & 54.92861 & 0 & $8.239708(-6)$ & 1 & $7.492530(2)$ & -1 & $-3.973338(5)$ & -2 & $1.522664(8)$ & -3 & $-2.489134(10)$ & - \\
\hline $\bar{\Omega}^{(2,2)}$ & $\mathrm{Ar}-\mathrm{Ar}^{+}$ & -2.463054 & 29.65574 & 0 & $1.826000(-5)$ & 1 & $3.562999(3)$ & & $-6.882292(5)$ & -2 & $-1.622056(8)$ & & $7.148037(10) \mid-4$ & - \\
\hline $\bar{\Omega}(1,1)$ & $\mathrm{Ar}-\mathrm{H}^{+}$ & - & 31.35736 & 0 & $-9.863792(-3)$ & 1 & $2.694942(-6)$ & 2 & $|-4.293073(-10)|$ & 3 & $3.699968(-14)$ & 4 & $-1.622317(-18) 5$ & $2.847661(-23) 6$ \\
\hline $\bar{\Omega}(2,2)$ & $\mathrm{Ar}-\mathrm{H}^{+}$ & - & 27.65083 & 0 & $-9.789488(-3)$ & 1 & $2.823633(-6)$ & 2 & $-4.386553(-10)$ & 3 & $3.635024(-14)$ & 4 & $-1.532922(-18)$ & $2.593524(-23) 6$ \\
\hline $\bar{\Omega}(1,1)$ & $\mathrm{Ar}^{+}-\mathrm{H}$ & 3.963178 & -28.03039 & 0 & $-4.885035(-4)$ & 1 & $3.905496(4)$ & -1 & $-3.975075(7)$ & -2 & $2.050230(10)$ & -3 & $-3.932595(12) \mid-4$ & - \\
\hline $\bar{\Omega}^{(2,2)}$ & $\mathrm{Ar}^{+}-\mathrm{H}$ & -1.070727 & 21.32955 & 0 & $-3.160291(-4)$ & 1 & $9.742487(3)$ & -1 & $-1.248955(7)$ & -2 & $7.616996(9)$ & -3 & $-1.631891(12) \mid-4$ & - \\
\hline $\bar{\Omega}(1,1)$ & $\mathrm{H}-\mathrm{H}^{+}$ & -3.903160 & 63.58838 & 0 & $1.586300(-5)$ & 1 & $1.442400(3)$ & -1 & $-7.648988(5)$ & $-2 \mid$ & $2.931197(8)$ & -3 & $-4.791625(10)$ & - \\
\hline $\bar{\Omega}^{(2,2)}$ & $H-H^{+}$ & -7.923776 & 79.11695 & 0 & $2.513210(-4)$ & 1 & $-1.943472(4)$ & -1 & $2.052439(7)$ & -2 & $-1.036902(10)$ & -3 & $1.962307(12)$ & - \\
\hline $\bar{\Omega}(1,1)$ & $\mathrm{H}_{2}$-chargée & -1.422830 & 15.80298 & 0 & $2.773230(-5)$ & 1 & $7.702305(3)$ & -1 & $-2.747496(6)$ & -2 & $9.549139(8)$ & -3 & $-1.496166(10) \mid-4$ & - \\
\hline $\bar{\Omega}^{(2,2)}$ & $\mathrm{H}_{2}$-chargée & -2.479067 & 27.53431 & 0 & $4.831933(-5)$ & 1 & $1.342011(4)$ & -1 & $-2.787100(6)$ & $-2 \mid$ & $1.663795(9)$ & -3 & $-2.606848(11)$ & - \\
\hline $\bar{Q}(1,1)$ & e-Ar & 0.1146081 & -0.7119644 & 0 & $3.128209(-4)$ & 1 & $-1.043603(3)$ & -1 & $1.503605(6)$ & -2 & $-6.185017(8)$ & -3 & $9.506717(10)$ & - \\
\hline $\bar{Q}(1,2)$ & e-Ar & 0.2475099 & -1.846789 & 0 & $4.071179(-4)$ & & $-1.495856(2)$ & $-1]$ & $1.149539(5)$ & -2 & $1.427086(8)$ & -3 & $-4.934132(10)$ & - \\
\hline $\bar{Q}(1,3)$ & e-Ar & - & -0.1027995 & 0 & $4.972708(-4)$ & 1 & $7.62075+(-8)$ & 2 & $-2.047266(-11)$ & 3 & $2.375452(-15)$ & 4 & $-1.280360(-19)$ & $2.609321(-24) 6$ \\
\hline$\overline{0}(1,4)$ & $e-A r$ & - & -0.2580061 & 0 & $8.399458(-4)$ & 1 & $-2.153590(-8)$ & 2 & $-2.023690(-12)$ & 3 & $6.088804(-16)$ & 4 & $-4.406308(-20) 5$ & $1.012320(-24) 66$ \\
\hline $\bar{Q}(1,5)$ & e-Ar & - & -0.3284474 & $0^{\prime}$ & $1.085572(-3)$ & 1 & $-8.260013(-8)$ & 2 & $9.571434(-12)$ & 3 & $-4.628693(-16)$ & 4 & $3.511461(-21)$ 5 & $1.642620(-25)$ \\
\hline $\bar{Q}^{(1,1)}$ & e-H & -9.571564 & 105.3011 & $0_{1}^{1}$ & $4.213696(-5)$ & 1 & $8.353974(3)$ & {$[-1$} & $-1.841851(7)$ & -2 & $1.057066(10)$ & -3 & $-2.081691(12)-4$ & - \\
\hline $\bar{\sigma}(1,2)$ & $\mathrm{e}-\mathrm{H}$ & -5.227312 & 62.35409 & & $-1.856845(-4)$ & 1 & $3.004984(4)$ & & $-3.316344(7)$ & $-2 \mid$ & $1.602566(10)$ & -3 & $-2.884492(12) \mid-4$ & - \\
\hline $\bar{Q}(1,3)$ & $e_{-H}$ & -3.418649 & 44.25786 & & $-3.200007(-4)$ & 1 & $3.405210(4)$ & -1 & $-3.059199(7)$ & -2 & $1.293895(10)$ & -3 & $-2.104117(12) \mid-4$ & - \\
\hline $\bar{Q}(1,4)$ & $e_{-H}$ & - & 43.43814 & $0^{\prime}$ & $'-1.278669(-2)$ & 1 & $2.822366(-6)$ & 2 & $\mid-3.830124(-10)$ & 3 & $2.975903(-14)$ & 4 & $-1.221989(-18) 5$ & $2.056622(-23)$ \\
\hline $\bar{D}(1,5)$ & $e_{-H}$ & - & 43.38269 & 0 & $-1.476739(-2)$ & 1 & $3.658660(-6)$ & 2 & $-5.449870(-10)$ & 3 & $4.567253(-14$ & 4. & $-1.995594(-18) 5$ & $3.536342(-23)$ \\
\hline $\bar{\nabla}(1,1)$ & $e_{-} \mathrm{H}_{2}$ & -3.556359 & 57.26338 & 0 & $-4.616636(-4)$ & 1 & $-4.746448(4)$ & d & $4.593245(7)$ & -2 & $-2.236737(10)$ & -3 & $4.126815(12)=-4$ & - \\
\hline$\overline{5}(1,2)$ & $\mathrm{e}-\mathrm{H}_{2}$ & -15.09241 & 160.9032 & 0 & $7.153855(-5)$ & & $-9.281039(4)$ & -1 & $7.944985(7)$ & -2 & $-3.648628(10)$ & -3 & $6.504251(12)-4$ & - \\
\hline$\overline{0}(1,3)$ & $e_{-} \mathrm{H}_{2}$ & -24.70968 & 244.7917 & 0 & $\Xi .473443(-4)$ & & $-1.221003(5)$ & -1 & $9.630548(7)$ & -2 & $-4.207784(10)$ & -3 & $7.257332(12)-4$ & - \\
\hline$\overline{5}(1,4)$ & $e-H_{2}$ & -30.28456 & 290.9263 & 0 & $1.085837(-3)$ & 1 & $-1.295500(5)$ & -1 & $9.357157(7)$ & -2 & $-3.837721(10)$ & -3 & $5.320889(12)-4$ & - \\
\hline $\bar{Q}(1,5)$ & $e_{-} \mathrm{H}_{2}$ & -31.73219 & 299.4667 & $=$ & $1.329351(-3)$ & & $-1.180260(5)$ & -1 & $7.539379(7)$ & $-2 \mid$ & $-2.782312(10)$ & -3 & $4.196457(12)-4$ & - \\
\hline
\end{tabular}

la molécule $\mathrm{H}_{2}$ ). Les coefficients que nous avons utilisés sont les suivants :

$$
D_{\mathrm{e}}=7,019 \mathrm{meV} ; \quad r_{\mathrm{m}}=2,615 \AA ; \quad \beta=1,895 \AA^{-1},
$$

le potentiel est en bon accord avec les résultats expérimentaux de Colgate et al. [68] pour les énergies de l'ordre de $1 \mathrm{eV}$ mais il diffère alors notablement de ceux de Le Roy et al. [69] et de Rulis et al. [70].

2.1.4 Interaction $\mathrm{H}-\mathrm{H}$. - Cette interaction étudiée depuis longtemps car c'est la plus simple, se fait suivant deux courbes de potentiel qui correspondent, l'une à l'état fondamental de $\mathrm{H}_{2}$ (état ${ }^{1} \Sigma$ ) l'autre à un état répulsif (état ${ }^{3} \Sigma$ ). Nous avons utilisé, dans les deux cas, les résultats de Kolos et al. [71-73].

a) Nous avons écrit l'état ${ }^{3} \Sigma$ sous la forme exponentielle répulsive avec :

$$
V_{0}=46,02 \mathrm{eV} ; \quad B=2,603 \AA^{-1} .
$$

b) Dans la littérature l'état ${ }^{1} \Sigma$ est ajusté par des formes analytiques simples, soit dans sa partie dispersive à longue distance $[74,75]$, soit dans la région du fond du puits de potentiel [76, 77]. Cependant dans la plage d'énergie qui nous intéresse, ces formes analytiques donnent une mauvaise approximation des résultats de Kolos et al. [71] ce qui fait que nous avons ajusté ce potentiel par deux formes :

- aux basses énergies, par un potentiel exponentiel attractif avec :

$$
V_{0}=-177,79 \mathrm{eV} ; \quad B=2,824 \AA^{-1},
$$

- aux hautes énergies, par un potentiel puissance attractif avec :

$$
V_{0}=-12,43 \mathrm{eV} \AA^{-4,36} ; n=4,36 .
$$

Remarquons que ce dernier potentiel est celui utilisé par Vanderslice et al. [78]. 
2.1.5 Interaction $\mathrm{H}-\mathrm{H}_{2}$. - De nombreux potentiels isotropes ont été développés (ils sont répertoriés en partie par Torello et al. [79]) pour la zone dispersive et le début de la zone répulsive. Pour la zone répulsive proprement dite on doit introduire une partie anisotrope [76,80], cependant on montre que jusqu'à une distance interparticulaire de $1 \AA$ la partie isotrope du potentiel est prépondérante. Nous avons donc utilisé le potentiel isotrope BMSV de Gengenback et al. [81] qui s'écrit pour la zone répulsive :

$V(r)=V_{0}\left(\exp (-B r)-\exp \left(-B r_{0}\right)\right)$ pour $r<3,4 \AA$,

où les coefficients sont les suivants :

$$
V_{0}=55,9 \mathrm{eV} ; \quad B=3,065 \AA^{-1} ; \quad r_{0}=3,14 \AA .
$$

Pour la détermination des intégrales de collision, nous avons réduit ce potentiel à sa partie variable, la suppression de la partie constante introduit une erreur maximale de $8 \%$ à $500 \mathrm{~K}$ et elle devient inférieure à $2 \%$ pour $T>1500 \mathrm{~K}$.

Ce potentiel expérimental jusqu'à $0,2 \mathrm{eV}$ est extrapolé pour les valeurs supérieures où il est en bon accord avec d'autres mais surtout avec celui de Liu [82].

2.1.6 Interaction $\mathrm{H}_{2}-\mathrm{H}_{2}$. - Pour cette interaction les potentiels diffèrent beaucoup [83-87]. Nous avons décidé d'utiliser le potentiel répulsif calculé de Ree et al. $[88,89]$ pour deux raisons : il peut être considéré comme une " moyenne " des résultats expérimentaux et il est en bon accord avec celui de Schaeffer et al. [90]. Pour sa partie isotrope (obtenue par intégration d'un potentiel anisotrope plus complexe) il s'écrit, en respectant les notations de Ree :

$$
V(r)=A(r)+\frac{2}{3} B(r)+\frac{D(r)}{9} .
$$

Nous l'avons transformé en un potentiel exponentiel répulsif avec :

$$
V_{0}=252,5 \mathrm{eV} ; \quad B=3,331 \AA^{-1} .
$$

2.1.7 Interaction $\mathrm{Ar}-\mathrm{Ar}^{+}$. - Cette interaction se fait suivant six courbes de potentiel, si l'on tient compte de couplage spin-orbite, correspondant aux états ${ }^{2} \Sigma_{1 / 2 \mathrm{u}},{ }^{2} \Pi_{3 / 2 \mathrm{~g}},{ }^{2} \Pi_{1 / 2 \mathrm{~g}},{ }^{2} \Pi_{3 / 2 \mathrm{u}},{ }^{2} \Pi_{1 / 2 \mathrm{u}}$ et ${ }^{2} \Pi_{1 / 2 \mathrm{~g}}$ de
$\mathrm{Ar}_{2}^{+}$, chaque état ayant un poids statistique de 2 . Pour nos calculs nous avons utilisés les potentiels expérimentaux de Moseley et al. [91] et ceux calculés de Michels et al. [92] (qui sont aussi en accord avec ceux de Stevens et al. [93]) qui tiennent compte du couplage spin-orbite.

2.1.7.1 Intégrales de type diffusion $(l=1)$. Pour ce cas de transfert résonnant, nous avons exprimés les différences de potentiel $\mathrm{g}-\mathrm{u}$ [52]

$$
\begin{aligned}
& \left|V\left({ }^{2} \Sigma_{\mathrm{g}}\right)-V\left({ }^{2} \Sigma_{\mathrm{u}}\right)\right|=240,33 \exp (-1,661 r), \\
& \left|V\left({ }^{2} \Pi_{\mathrm{g}}\right)-V\left({ }^{2} \Pi_{\mathrm{u}}\right)\right|=167,62 \exp (-2,254 r) .
\end{aligned}
$$

Pour les états $\Pi$ l'ajustement est fait pour la différence ${ }^{2} \Pi_{3 / 2 \mathrm{u}}-{ }^{2} \Pi_{3 / 2 \mathrm{~g}}$, ces deux derniers états ayant la même limite de potentiel.

Avec les relations simplifiées, nous avons déterminé les grandeurs nécessaires aux calculs des intégrales de collision :

$$
\begin{aligned}
& A_{\Sigma}=26,39 \AA ; \quad B_{\Sigma}=1,120 \AA ; \\
& A_{\Pi}=18,96 \AA ; \quad B_{\Pi}=0,8301 \AA .
\end{aligned}
$$

L'intégrale moyenne obtenue, après pondération par les poids statistiques ( 2 pour l'état $\Sigma$ et 4 pour l'état $\Pi$ ) est inférieure à celle utilisée par Devoto [34] aux basses températures $(5 \%$ à $3000 \mathrm{~K})$ et supérieure à hautes températures $(8 \%$ à $15000 \mathrm{~K})$.

2.1.7.2 Intégrale de type viscosité $(l=2)$. - Ces intégrales ne sont pas affectées par le transfert de charge et nous donnons les potentiels utilisés pour les différents états.

- Pour l'état ${ }^{2} \Sigma_{1 / 2 u}$ nous avons effectué un ajustement avec un potentiel de Morse ayant pour coefficients :

$$
D_{\mathrm{e}}=1,34 \mathrm{eV} ; \quad r_{\mathrm{m}}=2,43 \AA ; \quad \beta=1,69 \AA^{-1} .
$$

- Pour les états ${ }^{2} \Pi_{\mathrm{g}}$, compte tenu des incertitudes sur la position d'équilibre ( $r_{\mathrm{m}}$ compris entre 3 et $3,4 \AA$ ) et sur la profondeur du puits de potentiel $\left(D_{\mathrm{e}}\right.$ compris entre 0,25 et $0,06 \mathrm{eV}$ ) selon les auteurs [91-94], nous avons effectué les ajustements sur les parties répulsives qui sont en meilleur accord. Les coefficients du potentiel de Morse sont :

$$
\begin{array}{llll}
\text { Etat }^{2} \Pi_{3 / 2 \mathrm{~g}} & D_{\mathrm{e}}=0,21 \mathrm{eV} ; & r_{\mathrm{m}}=3,077 \AA ; & \beta=1,629 \AA^{-1} . \\
\text { Etats }^{2} \Pi_{1 / 2 \mathrm{~g}} & D_{\mathrm{e}}=0,10 \mathrm{eV} ; & r_{\mathrm{m}}=3,161 \AA ; & \beta=1,795 \AA^{-1}
\end{array}
$$

- Pour les états ${ }^{2} \Pi_{\mathrm{u}}$, et ${ }^{2} \Sigma_{1 / 2 \mathrm{~g}}$, nous avons utilisé des potentiels exponentiels répulsifs avec :

$$
\begin{array}{llll}
\text { Etat }^{2} \Pi_{3 / 2 \mathrm{u}} & V_{0}=2,685 \times 10^{6} \mathrm{eV} ; & B=5,889 \AA^{-1} \quad & \text { (basse énergie) } \\
& V_{0}=29106 \mathrm{eV} ; & B=4,154 \AA^{-1} \quad \text { (haute énergie) } \\
\text { Etat }^{2} \Pi_{1 / 2 \mathrm{u}} & V_{0}=1,655 \times 10^{4} \mathrm{eV} ; & B=3,880 \AA^{-1} . & \\
\text { Etat }^{2} \Sigma_{1 / 2 \mathrm{~g}} & V_{0}=369,0 \mathrm{eV} ; & B=2,031 \AA^{-1} .
\end{array}
$$

L'intégrale de collision moyenne est obtenue par pondération sur les différents états. 
2.1.8 Interaction $\mathrm{Ar}-\mathrm{H}^{+}$. - Pour cette interaction beaucoup étudiée vers les années 1970 [95-99], nous avons retenu le potentiel de Sidis [99] modifié pour tenir compte de résultats expérimentaux (ce dernier est d'ailleurs conseillé dans une publication plus récente [100]). Nous l'avons exprimé sous la forme d'un potentiel de Morse avec :

$$
D_{\mathrm{e}}=4,1 \mathrm{eV} ; \quad r_{\mathrm{m}}=1,33 \AA ; \quad \beta=1,792 \AA^{-1} .
$$

2.1.9 Interaction $\mathrm{H}-\mathrm{Ar}^{+}$. - Pour cette collision nous avons tenu compte de quatre états répulsifs (qui sont les ${ }^{3} \Sigma,{ }^{1} \Pi,{ }^{3} \Pi$ et ${ }^{1} \Sigma$ ) calculés théoriquement par Kuntz et al. [95] et ayant pour poids statistique, respectivement $3,2,6$ et 1 . Nous faisons des ajustements par des potentiels répulsifs (exponentiel ou puissance) mais, sur l'intervalle qui nous intéresse nous sommes obligés de les faire en deux ou trois parties :

\begin{tabular}{|c|c|c|c|}
\hline Etat $^{3} \Sigma$ & $\begin{array}{l}V_{0}=3,599 \times 10^{4} \mathrm{eV} \\
V_{0}=961,8 \mathrm{eV}\end{array}$ & $\begin{array}{l}B=5,154 \AA^{-1} \\
B=3,537 \AA^{-1}\end{array}$ & $\begin{array}{l}\text { (basse énergie) } \\
\text { (haute énergie). }\end{array}$ \\
\hline Etat $^{1} \Pi$ & $\begin{array}{l}V_{0}=1,028 \times 10^{4} \mathrm{eV} ; \\
V_{0}=101 \mathrm{eV} ; \\
V_{0}=10,801 \mathrm{eV} \AA^{-2,051} ;\end{array}$ & $\begin{array}{l}B=2,628 \AA^{-1} \\
B=1,454 \AA^{-1} \\
n=2,051\end{array}$ & $\begin{array}{l}\text { (basse énergie) } \\
\text { (moyenne énergie) } \\
\text { (haute énergie). }\end{array}$ \\
\hline Etat ${ }^{3} \Pi$ & $\begin{array}{l}V_{0}=1,028 \times 10^{4} \mathrm{eV} \\
V_{0}=73,31 \mathrm{eV} \\
V_{0}=7,946 \mathrm{eV} \AA^{-1,919}\end{array}$ & $\begin{array}{l}B=2,648 \AA^{-1} \\
B=1,393 \AA^{-1} \\
n=1,919\end{array}$ & $\begin{array}{l}\text { (basse énergie) } \\
\text { (moyenne énergie) } \\
\text { (haute énergie). }\end{array}$ \\
\hline $\operatorname{Etat}^{1} \Sigma$ & $\begin{array}{l}V_{0}=5,317 \times 10^{5} \mathrm{eV} \\
V_{0}=6,774 \times 10^{3} \mathrm{eV} \\
V_{0}=303,4 \mathrm{eV}\end{array}$ & $\begin{array}{l}B=3,386 \AA^{-1} \\
B=2,374 \AA^{-1} \\
B=1,556 \AA^{-1}\end{array}$ & $\begin{array}{l}\text { (basse énergie) } \\
\text { (moyenne énergie) } \\
\text { (haute énergie) }\end{array}$ \\
\hline
\end{tabular}

2.1.10 Interaction $\mathrm{H}-\mathrm{H}^{+}$. - Cette interaction se fait suivant deux courbes de potentiel correspondant aux états ${ }^{2} \Sigma_{\mathrm{u}}$ et ${ }^{2} \Sigma_{\mathrm{g}}$ (chacun de poids statistique 2) de $\mathrm{H}_{2}^{+}$et comme dans le cas de la collision $\mathrm{Ar}-\mathrm{Ar}^{+}$ nous découplons le calcul de $\bar{\Omega}^{(l, 5)}$ de celui de $\bar{\Omega}^{(2,2)}$. Nous utilisons les potentiels de Kuntz et al. [95] et de Peek [101].

2.1.10.1 Intégrales de type diffusion $(l=1)$. Nous exprimons la différence de potentiel $\mathrm{g}-\mathrm{u}$ comme suit [52] :

$$
\left|V\left(\Sigma_{\mathrm{g}}\right)-V\left(\Sigma_{\mathrm{u}}\right)\right|=58,27 \exp (-1,464 r) .
$$

Puis la résolution de l'équation transcendante simplifiée et après ajustement, nous obtenons les coefficients nécessaires aux calculs des intégrales de collision :

$$
A_{\Sigma}=28,69 \AA ; \quad B_{\Sigma}=1,300 \AA .
$$

2.1.10.2 Intégrale de type viscosité $(l=2)$. Pour l'état répulsif $\Sigma_{\mathrm{u}}$ nous ajustons les données de Peek [101] pour un potentiel exponentiel avec :

$$
V_{0}=51,75 \mathrm{eV} ; \quad B=1,677 \AA^{-1} .
$$

Pour l'état attractif $\Sigma_{\mathbf{g}}$ nous avons des potentiels puissances (la basse énergie étant celui de Belov [102]) avec :

$V_{0}=-282 \mathrm{eV} \AA^{-5,8} ; \quad n=5,8 \quad$ (basse énergie)

$V_{0}=-18,76 \mathrm{eV} \AA^{-3,47} ; n=3,47 \quad$ (haute énergie).
2.1.11 Interactions $\mathrm{H}_{2}-\mathrm{Ar}^{+}$et $\mathrm{H}_{2}-\mathrm{H}^{+}$. - La contribution de ces interactions est faible, nous les avons représentées par des potentiels de polarisation, la polarisabilité de $\mathrm{H}_{2}$ étant [103] :

$$
\alpha_{\mathrm{H}_{2}}=0,767 \AA^{2} .
$$

Nous écrivons le potentiel sous la forme suivante :

$$
V(r)=-5,522 r^{-4} .
$$

2.1.12 Interaction électron-neutre. - Les intégrales de collision $(l=1)$ se calculent par intégration numérique (méthode de Gauss-Laguerre à 32 points) à partir de la relation de définition de $\bar{\Omega}^{(l, s)}$. Nous avons utilisé les sections efficaces de transfert $Q^{i}$ fournies par Itikawa $[104,105]$ que nous avons complétées. Pour la collision e-Ar nous avons extrapolé $Q^{(l)}$ à partir des résultats de Frost et al. [106].

Pour la collision e- $\mathrm{H}_{2}$ nous avons interpolé entre les résultats d'Itikawa qui s'arrêtent à $10 \mathrm{eV}$ et ceux de Kupta et al. [107] qui débutent à $100 \mathrm{eV}$.

2.2 Résultats. - 2.2.1 Composition. - La composition du plasma argon-hydrogène à l'ETLC est calculée par la méthode décrite précédemment, de $500 \mathrm{~K}$ à $15000 \mathrm{~K}$ par pas de $100 \mathrm{~K}$ et à la pression atmosphérique. Les énergies libres molaires sont obtenues à partir des fonctions de partition de Capitelli et al. [25] que nous avons complétées jusqu'à $500 \mathrm{~K}$ grâce aux tables de la JANAF. Sur notre plage de température, les fonctions de partition sont indépen- 
dantes du rapport $\mathrm{H}_{2} / \mathrm{Ar}$, nous avons fait un ajustement (sur 16 points) par espèce chimique.

Nos résultats, obtenus avec la longueur de Debye totale (c'est-à-dire en tenant compte des ions) lors de la détermination de l'abaissement du potentiel d'ionisation et de pression, correspondent avec ceux de Capitelli et al. [25] à moins de $1 \%$ pour les mélanges $\mathrm{Ar}-\mathrm{H}_{2}$ à la pression atmosphérique. Ils sont aussi en très bon accord avec ceux de Fauchais [108] et de Patch [109] pour l'hydrogène pur. Les propriétés de transport sont calculées pour cinq valeurs du rapport $\mathrm{H}_{2} /\left(\mathrm{H}_{2}+\mathrm{Ar}\right)$ : de l'hydrogène pur à l'argon pur par saut de $25 \%$. Les courbes correspondantes sont référencées respectivement de 1 (hydrogène pur) à 5 (argon pur) sur les figures.

2.2.2 Propriétés de transport. - Nos résultats sont comparés, en général, avec ceux de Gorse [18] pour lesquels nous possédons un maximum d'informations.

2.2.2.1 Viscosité. - Sur la figure 1 sont comparées la viscosité de l'argon obtenue théoriquement par Gorse [10], Devoto [52] et nous-mêmes ainsi que celles dérivées de données expérimentales [123] jusqu’à $2500 \mathrm{~K}$. Nos résultats sont en bon accord avec ces dernières (à moins de $2 \%$ sur l'ensemble de la plage), ceux de Gorse sont, à $2000 \mathrm{~K}, 10 \%$ supérieurs. Ceci montre, du moins aux basses températures, que l'intégrale $\bar{\Omega}^{(2,2)}$ de la collision Ar-Ar déterminée par Gorse est trop faible de $10 \%$ (la viscosité évoluant en raison inverse de $\bar{\Omega}^{(2,2)}$ ). A haute température, l'intégrale $\bar{\Omega}^{(2,2)}$ de la collision $\mathrm{Ar}^{+}-\mathrm{Ar}^{+}$devient dominante et nos résultats concordent avec ceux de Gorse (nous calculons $\bar{\Omega}^{(2,2)}$ à partir du même potentiel). Les viscosités, correspondant aux cinq compositions, sont représentées sur la figure 2. Aux basses

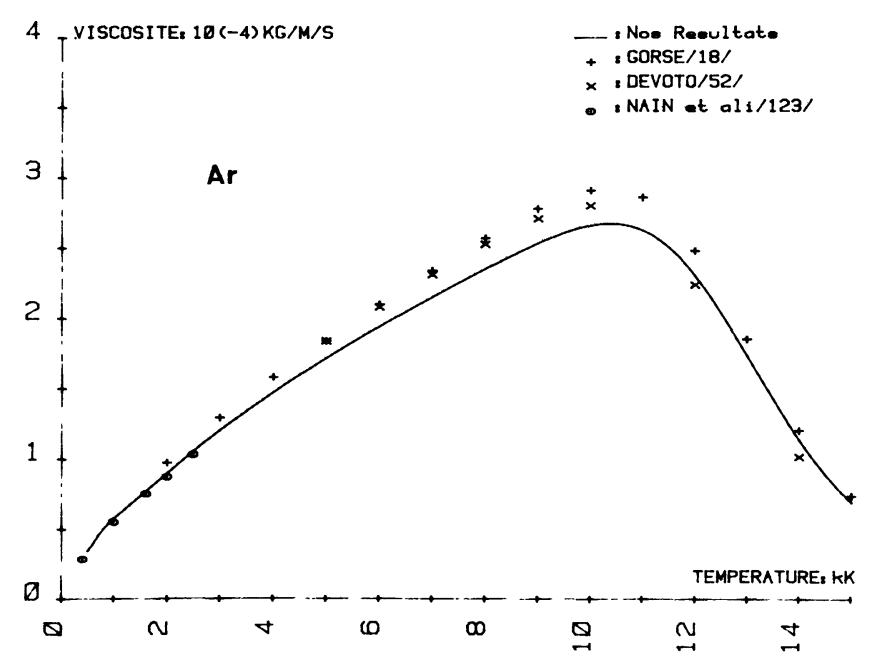

Fig. 1. - Comparaison de la viscosité de l'argon en fonction de la température et à la pression atmosphérique entre différents auteurs.

[Evolution of the argon viscosity with temperature (1 atm.). Comparison between the results obtained by various authors.]

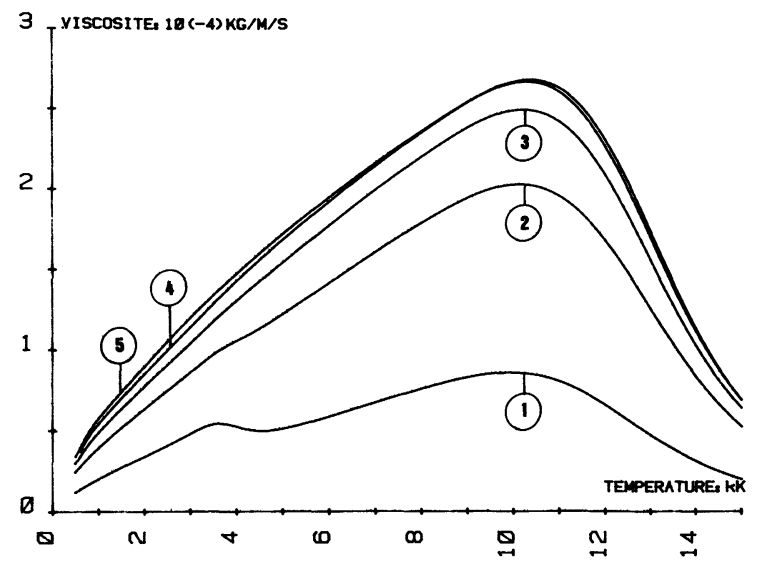

Fig. 2. - Viscosité en fonction de la température, pour les cinq valeurs du rapport $\mathrm{H}_{2} /\left(\mathrm{H}_{2}+\mathrm{Ar}\right)$ à la pression atmosphérique.

[Evolution of the viscosity with temperature for five values of the $\mathrm{H}_{2} / \mathrm{H}_{2}+\mathrm{Ar}$ ) ratio (1 atm.).]

températures, nos résultats sont, en général, inférieurs à ceux de Gorse (nos valeurs de l'intégrale $\bar{\Omega}^{(2,2)}$ des collisions $\mathrm{Ar}-\mathrm{Ar}$ et $\mathrm{Ar}-\mathrm{H}_{2}$ étant supérieures aux siennes) à l'exception de celle de l'hydrogène pur $\left(7 \%\right.$ en plus à $2000 \mathrm{~K}$, l'intégrale $\bar{\Omega}^{(2,2)}$ pour la collision $\mathrm{H}_{2}-\mathrm{H}_{2}$ est alors de $7 \%$ inférieure). Aux hautes températures, les collisions ion-ion deviennent dominantes, les résultats sont alors en bon accord, l'écart relatif est de l'ordre de $2 \%$ à $15000 \mathrm{~K}$, quelle que soit la composition.

2.2.2.2 Conductibilité thermique. - C'est la somme de quatre composantes :

$$
\lambda_{\mathrm{T}}=\left(\lambda_{\mathrm{tr}}^{\mathrm{h}}+\lambda_{\mathrm{tr}}^{\mathrm{e}}\right)+\lambda_{\mathrm{I}}+\lambda_{\mathrm{R}} .
$$

Nous avons représenté sur la figure 3 la contribution de ces quatre conductibilités pour l'hydrogène pur.

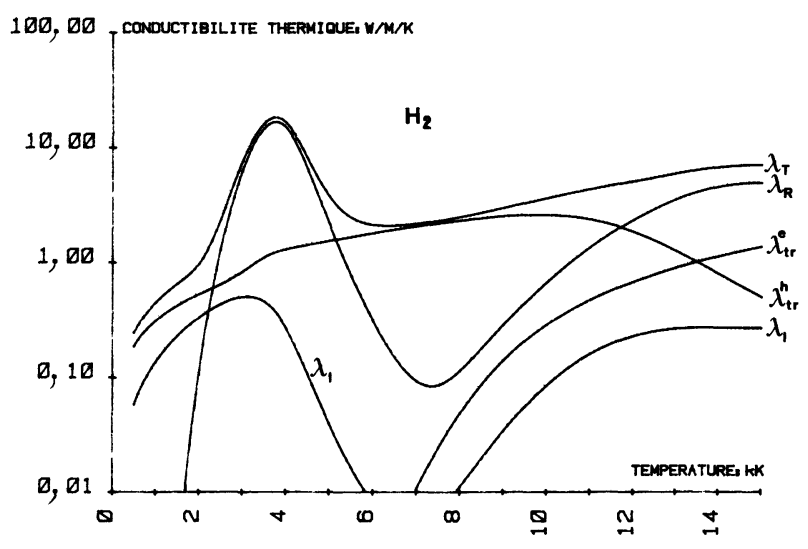

Fig. 3. - Conductibilité thermique totale ainsi que ses quatre composantes, en fonction de la température, pour l'hydrogène (1 atm.).

[Evolution with temperature of the total thermal conductivity and its four components, for an hydrogen plasma (1 atm.).] 
Pour notre plage de température, les deux contributions dominantes sont les conductibilités de translation des particules lourdes et de réaction, leur somme représente toujours plus de $75 \%$ de $\lambda_{\mathrm{T}}$ et elle est souvent supérieure à $90 \%$ de $\lambda_{\mathrm{T}}$. La conductibilité de translation des électrons n'est jamais importante : au maximum $20 \%$ de $\lambda_{\mathrm{T}}$ à $15000 \mathrm{~K}$; cependant, audelà de $15000 \mathrm{~K}$, sa part augmenterait rapidement avec la température. Quant à la conductibilité interne, elle est toujours faible, sa plus grande contribution relative (par rapport à $\lambda_{\mathrm{T}}$ ) est de $25 \%$ à basse température (vers $2000 \mathrm{~K}$ ) et elle est, en général, de l'ordre du pour cent. La conductibilité thermique passe par un maximum $\left(\lambda_{\mathrm{T}}=18,3 \mathrm{~W} / \mathrm{mK}\right)$ vers $3800 \mathrm{~K}$ alors que Gorse situe le maximum de son pic, lié à la dissociation de l'hydrogène moléculaire, à $4000 \mathrm{~K}$ avec $\lambda_{\mathrm{T}}=15 \mathrm{~W} / \mathrm{mK}$. Nous expliquons cette différence sur la position du sommet du pic par le fait que Gorse utilise les tables de Capitelli et al. [25] dans lesquelles la composition est donnée par pas de $1000 \mathrm{~K}$ et qu'ainsi elle a « raté » le maximum de la conductibilité thermique. Cela justifie l'utilisation d'un programme de calcul de la composition en amont du programme de détermination des propriétés de transport. Cependant, en dehors du pic de dissociation, nos résultats sont en très bon accord avec ceux de Gorse, l'erreur relative étant inférieure à $1 \%$ pour les températures supérieures à $5000 \mathrm{~K}$. Signalons finalement que la conductibilité thermique est multipliée par 75 lorsque la température passe de $500 \mathrm{~K}$ à $3800 \mathrm{~K}$.

Sur la figure 4 est représentée l'évolution de la conductibilité thermique totale des divers mélanges. On constate une évolution vers les basses températures du sommet du pic de dissociation de l'hydrogène : vers $3800 \mathrm{~K}$ pour l'hydrogène pur et seulement vers $3500 \mathrm{~K}$ pour la composition à $25 \%$ d'hydrogène, ce qui est logique compte tenu d'une dilution de plus en plus grande de l'hydrogène dans l'argon. Dans le

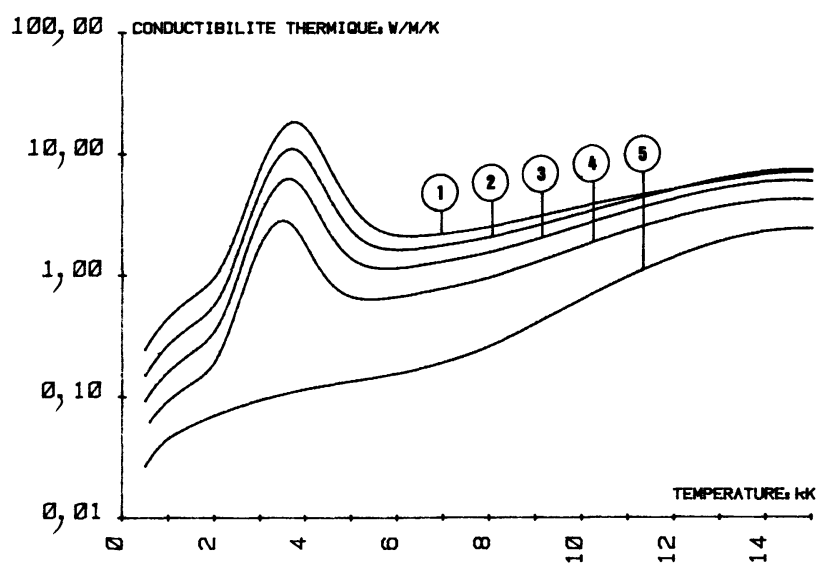

Fig. 4. - Conductibilité thermique totale, en fonction de la température, pour les cinq valeurs du rapport $\mathrm{H}_{2} /\left(\mathrm{H}_{2}+\mathrm{Ar}\right)$ et à la pression atmosphérique.

[Evolution with temperature of the total thermal conductivity for five values of the $\mathrm{H}_{2} /\left(\mathrm{H}_{2}+\mathrm{Ar}\right)$ ratio $(1 \mathrm{~atm}$.).] cas de l'argon pur, nos résultats sont toujours inférieurs à ceux de Gorse (de $8 \%$ à $2000 \mathrm{~K}$ ) du fait de nos intégrales supérieures pour la collision Ar-Ar, mais ils sont en meilleur accord avec les résultats expérimentaux comme pour la viscosité.

2.2.2.3 Conductivité électrique. - Sur la figure 5, nous représentons la conductivité électrique pour l'argon et l'hydrogène, les autres compositions donnent des résultats intermédiaires. Nous rappelons que, lors du calcul des intégrales de collision entre particules chargées (particulièrement importante pour cette grandeur) nous avons utilisé la longueur de Debye réduite alors que Gorse prend en compte la contribution des ions. Malgré cette différence de choix, nos résultats sont toujours en accord avec ceux de Gorse, aussi bien pour l'hydrogène que pour l'argon, lorsque la conductivité électrique devient une réalité physique $(T>6000 \mathrm{~K}): 5 \%$ à $7000 \mathrm{~K}$ et $2 \%$ à $15000 \mathrm{~K}$ d'erreur relative pour l'argon et toujours moins de $2 \%$ d'erreur relative pour l'hydrogène. L'erreur plus importante à basse température pour l'argon est liée à la collision e-Ar, les sections efficaces de transfert $Q^{(l)}$ utilisées diffèrent notablement aux basses énergies (au niveau de l'effet Ramsauer), les valeurs de $Q^{(l)}$ retenues par Gorse [104] ayant évolué [105].

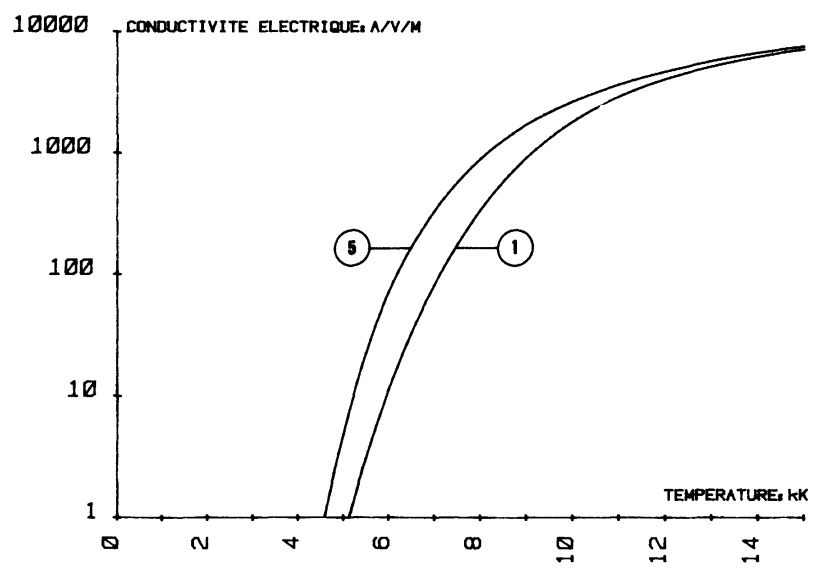

Fig. 5. - Conductivité électrique, en fonction de la température, pour l'hydrogène (1) et l'argon (5), à la pression atmosphérique.

[Evolution with temperature of the electrical conductivity for hydrogen (1) and argon (5) plasma respectively (1 atm.).]

3. Conclusion. - Avec le développement croissant de l'utilisation des plasmas thermiques : four à plasma pour la métallurgie extractive, pour la refusion des métaux, sphéroïdisation ou projection de particules céramiques ou métalliques, il semblait nécessaire de reprendre le calcul des propriétés de transport des plasmas, calculs dont la majorité a été effectuée avant 1976.

En effet, ces dernières années les progrès de la 
physique atomique ont permis de préciser, soit expérimentalement, soit théoriquement les potentiels d'interaction et il convenait de voir dans quelle mesure leurs nouvelles valeurs modifient les propriétés de transport. Après une revue générale des différents types de potentiels, nous avons rassemblé ceux relatifs au mélange $\mathrm{Ar}-\mathrm{H}_{2}$, un des plus utilisé dans la pratique, soit en conservant les mieux adaptés compte tenu des données expérimentales, soit en ajustant différents potentiels sur les parties où l'accord est le meilleur et nous avons calculé les intégrales de collision cor- respondantes résumées ici sous forme de polynômes. Par rapport aux calculs antérieurs les écarts sur les intégrales de collision varient entre 1 et $10 \%$ en moyenne. Les propriétés de transport qui s'en déduisent sont en assez bon accord avec les valeurs déterminées avant 1976 avec des écarts qui ne dépassent pas $10 \%$. Cependant, à des températures de 500 à $5000 \mathrm{~K}$, la notion d'équilibre thermodynamique local perd son sens; c'est pourquoi nous développons des calculs de propriétés de transport hors équilibre à deux températures électroniques et des particules lourdes.

Bibliographie

[1] WALdie, B., 4th International Symposium on Plasma Chemistry (Zurich) août 1979.

[2] Schoumaker, H. R. P., Conference Proceedings of International Round Table on Study and Application of Transport Phenomena in Thermal Plasma (Odeillo) septembre 1975.

[3] Rykalin, N. N., Kudinov, V. V., Pure Appl. Chem. 48 (1976) 229.

[4] Vardelle, M., Vardelle, A., Besson, J. L., FauCHAIs, P., Revue Phys. Appl. 16 (1981) 425.

[5] Mac Rae, D. R., Gold, R. G., Thomson, C. D., SANDALL, W. R., 3th International Symposium on Plasma Chemistry (Limoges) juillet 1977.

[6] Pateyron, B., Aubreton, J., Kassabji, F., FauCHAIS, P., 5th International Symposium on Plasma Chemistry, ed. B. Waldie and G. A. Farnell (Edinburgh) août 1981.

[7] MoNK, J. R., 5th International Symposium on Plasma Chemistry, Ed. B. Waldie and G. A. Farnell (Edinburgh) août 1981.

[8] Fey, M. G., Meyer, T. N., Reed, W. H., 4th International Symposium on Plasma Chemistry (Zurich) août 1979.

[9] Fey, M. G., Harvey, F. J., Met. Eng. Q. mai 1976.

[10] Fey, M. G., MeliLli, W. J., 5th International Symposium on Plasma Chemistry. Ed. B. Waldie and G. A. Farnell (Edinburgh) août 1981.

[11] Santen, S., International Industrial Seminar on Pilot Plan Experiences (Melting and Processing Technology, Seabrook Island, South Carolina) octobre 1980.

[12] Aakesson, J., International Industrial Seminar in Pilot Plant Experiences (Melting and Processing Technology, Seabrook Island, South Carolina) octobre 1980.

[13] Yamaguchi, K., International Industrial Seminar in Pilot Plan Experiences (Melting and Processing Technology, Seabrook Island, South Carolina) octobre 1980.

[14] Gauvin, W. H., KubaneK, G. R., Irons, G. A., J. Met. 33 (1981) 42.

[15] YAMAGUCHI, K., International Industrial Seminar on Pilot Plan Experiences (Melting and Processing Technology, Seabrook Island, South Carolina) octobre 1980.
[16] Bourdin, E., Fauchais, P., Boulos, M., National Heat Transfer Symposium (Edmonton, Alberta) octobre 1980, soumis à Int. J. Heat Mass Transfer.

[17] IUPAC sub committee on plasma chemistry, soumis à Pure Appl. Chem.

[18] Gorse, C., Thèse 3e cycle, Limoges (1975).

[19] Abdelhakim, H., Dinguirard, J. P., Vacquie, S., J. Phys. D : Appl. Phys. 13 (1980) 1427.

[20] Dinulescu, H., Pfender, E., Wilhemi, H., 5th International Symposium on Plasma Chemistry, Ed. B. Waldie and G. A. Farnell (Edinburgh) août 1981.

[21] White, W. B., Johnson, S. M., Dantzig, G. B., J. Chem. Phys. 28 (1958) 751.

[22] Griem, H. R., Plasma Spectroscopy (McGraw-Hill Book Company) 1964, p. 138.

[23] Drawin, H. W., Reactions under Plasma Conditions Vol. 1, Ed. by M. Venugopalan (Wiley-Interscience) 1971, p. 66.

[24] Fauchais, P., 2nd World Congress of Chemical Engineering and World Chemical Exposition, Plasma : Technology and Applications, vol. 1, (Montreal) octobre 1981, p. 1.58.

[25] Capitelli, M., Ficocelli, E., Molinari, E., Equilibrium Compositions and Thermodynamic Properties of Mixed Plasmas (Bari) juillet 1972.

[26] Bayard, S., Thèse 3e cycle, Limoges (1974).

[27] Fauchais, P., Baronnet, J. M., Bayard, S., Rev. Int. Hautes Temp. et Refract. 12 (1975) 221.

[28] Bonnefor, C., Thèse 3e cycle, Limoges (1975).

[29] Devoto, R. S., Phys. Fluids 9 (1966) 1230.

[30] Devoto, R. S., Phys. Fluids 10 (1967) 2105.

[31] Hirschfelder, J. D., Curtiss, C. F., BiRd, R. B., Molecular Theory of Gases and Liquids (John Wiley) 1954, p. 486.

[32] Liboff, R. L., Phys. Fluids 2 (1959) 40.

[33] Mason, E. A., Munn, R. J., Smith, F. J., Phys. Fluids 10 (1967) 1827.

[34] Devoto, R. S., Phys. Fluids 16 (1973) 616.

[35] Kihara, T., Taylor, M. H., Hirschfelder, J. O., Phys. Fluids 3 (1960) 715.

[36] Monchick, L., Phys. Fluids 2 (1959) 695.

[37] BroKaw, R. S., Phys. Fluids 4 (1961) 944.

[38] Morse, P., Phys. Rev. 34 (1929) 57. 
[39] Samoilov, E. V., Tsitelauri, N. N., High Temp. 2 (1964) 509.

[40] Samoilov, E. V., Tsitelauri, N. N., High Temp. 7 (1969) 155.

[41] Sмith, F. J., Munn, R. J., J. Chem. Phys. 41 (1961) 3560 .

[42] Lennards-Jones, J. E., Proc. R. Soc. London A 106 (1924) 463.

[43] Warskel, A., Lifson, S., J. Chem. Phys. 53 (1970) 582.

[44] Ermer, O., Lifson, S., J. Am. Chem. Soc. 95 (1973) 443.

[45] Klein, M., SMith, F. J., J. Res. Nat. Bur. Stand. Sect A 72 (1968) 359.

[46] Neufeld, P. D., Janzen, A. R., Aziz, R. A., J. Chem. Phys. 57 (1972) 1100.

[47] Buckimgham, R. A., Proc. R. Soc. London A 168 (1938) 264.

[48] Mason, E. A., J. Chem. Phys. 22 (1954) 169.

[49] Monchick, L., Mason, E. A., J. Chem. Phys. 35 (1961) 1676

[50] Mason, E. A., Vanderslice, J. T., Yos, J. M., Phys. Fluids 6 (1959) 688.

[51] Dalgarno, A., Phil. Trans. R. Soc. London A 250 (1958) 426

[52] Devoto, R. S., Phys. Fluids 10 (1967) 354.

[53] Dalgarno, A., McDowell, M. R. C., Proc. $R$. Soc. A 69 (1956) 615.

[54] Bates, D. R., Boyd, A. H., Proc. Phys. Soc. London 80 (1962) 130.

[55] Van Brunt, V., Hsu, H. W., J. Non Equilib. Thermodyn. 3 (1978) 123.

[56] Linder, B., Hirshfelder, J. O., J. Chem. Phys. 28 (1958) 197.

[57] Parson, J. M., Siska, P. E., Lee, Y. T., J. Chem. Phys. 56 (1972) 1511.

[58] Clifford, A. A., Grey, P., Platts, N., J. Chem. Soc. Faraday Trans. I 73 (1977) 381.

[59] Rol, P., Communication privée citée par Azız, R. A., Chen, H. H., J. Chem. Phys. 67 (1977) 5719.

[60] Amour, I., Mason, E. A., J. Chem. Phys. 22 (1954) 670.

[61] Ahlrichs, R., Penco, P., Scoles, G., Chem. Phys. 19 (1976) 119.

[62] Bickes, R. W., Lantzsch, B., Toennies, J. P., Walaschewski, K., Discuss. Faraday Soc. 55 (1973) 167.

[63] Bassi, D., Dondi, M. G., Tomasini, F., Torello, F., Valbusa, U., Phys. Rev. A 13 (1976) 582.

[64] Tang, K. T., Toennies, J. P., J. Chem. Phys. 66 (1977) 1496.

[65] Das, G., Wagner, A. F., Wahl, A. C., J. Chem. Phys. 68 (1978) 4917.

[66] Brual, G., Rothstein, S. M., Chem. Phys. Lett. 61 (1979) 167.

[67] Dunker, A. M., Gordon, R. G., J. Chem. Phys. 68 (1978) 700.

[68] Colgate, S. O., Jordan, J. E., Mason, E. A., J. Chem. Phys. 51 (1969) 968.

[69] Le Roy, R. J., Conley, J. S., Grabenstetter, J. E., J. Chem. Soc. Faraday Discuss. 62 (1977) 169.

[70] Rulis, A. M., Smith, K. M., Scoles, G., Can. J. Phys. 56 (1978) 753.

[71] Kolos, W., Wolniewez, L., J. Chem. Phys. 43 (1965) 2429.
[72] Kolos, W., Wolniewez, L., J. Chem. Phys. 49 (1969) 404.

[73] Kolos, W., Wolniewez, L., J. Chem. Phys. Lett. 24 (1974) 457.

[74] Tang, K. T., Norbeck, J. M., Certain, P. R., J. Chem. Phys. 64 (1976) 3063.

[75] Yousif, F. N., Schukla, G. C., J. Chem. Phys. 70 (1979) 314.

[76] Howard, R. E., Yates, A. C., J. Chem. Phys. 66 (1977) 1960.

[77] Olson, J. A., MickA, D. A., J. Chem. Phys. 68 (1978) 4352.

[78] Vanderlice, J. T., Weismann, S., Mason, E. A., Fallen, R. J., Phys. Fluids 5 (1962) 155.

[79] Torello, F., Dondi, M. G., Chem. Phys. Lett. 55 (1978) 339.

[80] Truklar, D. G., Horowitz, C. J., J. Chem. Phys. 68 (1978) 2466.

[81] Gengenback, R., Hahn, Ch., Toennies, J. P., J. Chem. Phys. 62 (1975) 3620.

[82] Liu, B., J. Chem. Phys. 58 (1973) 1925.

[83] Farrar, J. M., Lee, Y. T., J. Chem. Phys. 57 (1972) 5492.

[84] Zarur, G., Rabitz, H., J. Chem. Phys. 60 (1974) 2057.

[85] Gallys, G. A., J. Chem. Phys. 66 (1977) 2252.

[86] Ramasuwamy, R., Rabitz, Green, S., J. Chem. Phys. 66 (1977) 3021.

[87] Starr, T. L., Williams, D. E., J. Chem. Phys. 66 (1977) 2054.

[88] Ree, F. H., Bender, C. F., J. Chem. Phys. 71 (1979) 5362.

[89] ReE, F. H., Bender, C. F., J. Chem. Phys. 73 (1980) 4712.

[90] Schaefer, J., Meyer, W., J. Chem. Phys. 70 (1979) 344.

[91] Moseley, J. T., Saxon, R. P., Huber, B. A., Cosby, P. C., Abouaf, R., Tadjeddine, M., J. Chem. Phys. 67 (1977) 1659.

[92] Michels, H. H., Hobbs, R. B., Wright, W. A., J. Chem. Phys. 69 (1978) 5151.

[93] Stevens, W. J., Gardner, M., Kano, A., Julienne, P., J. Chem. Phys. 67 (1977) 2860.

[94] Wadt, W. R., J. Chem. Phys. 68 (1978) 402

[95] Kuntz, P. J., Roach, A. C., J. Chem. Soc. Faraday Trans. II 68 (1972) 259.

[96] Champion, R. L., Doverspike, L. D., Rich, W. G., Bobilo, S. M., Phys. Rev. A 2 (1970) 2327.

[97] Rich, W. G., Bobbio, S. M., Champion, R. L., Doverspike, L. D., Phys. Rev. A 4 (1971) 2253.

[98] Klingbeil, R., J. Chem. Phys. 57 (1972) 1066.

[99] Sidis, V., J. Phys. B 5 (1972) 517.

[100] Matcha, R. L., Milleur, M. B., J. Chem. Phys. 69 (1978) 3016.

[101] Peek, J. M., J. Chem. Phys. 43 (1965) 3004.

[102] Belov, V. A., Teplofiz. Vys. Temp. 5(1967) 37.

[103] Kolos, W., Wolniewics, L., J. Chem. Phys. 46 (1967) 1426.

[104] ItIKawa, Y., At. Data Nucl. Data Tables 14 (1974) 1.

[105] Itikawa, Y., At. Data Nucl. Data Tables 21 (1978) 69.

[106] Frost, L. S., Phelps, A. V., Phys. Rev. 136 (1964) 1538.

[107] Kupta, P., Khare, S. P., J. Chem. Phys. 68 (1978) 2193.

[108] FaUchaIs, P., Thèse, Poitiers (1968). 
[109] PAтch, R. W., J. Quant. Spectrosc. Radiat. Transfer 9 (1969) 63.

[110] Vargaftik, N. B., Hemisphere Publishing Corporation, Washington-London (1975).

[111] Maitland, G. C., Smith, E. B., Chem. Phys. Lett. 22 (1973) 443.

[112] Varskni, Y. P., Rev. Mod. Phys. 29 (1957) 664.

[113] Keil, M., Kuppermann, A., J. Chem. Phys. 69 (1978) 3917.

[114] Klein, M., Hanley, H. J. M., J. Chem. Phys. 53 (1970) 4722.

[115] Hepburn, J., Scoles, G., Penco, R., Chem. Phys. Lett. 36 (1975) 451.
[116] Smith, K. M., Rulis, A. M., Scoles, G., Aziz, R. A., NaIn, V., J. Chem. Phys. 67 (1977) 152.

[117] Barker, J. A., Pompe, A., Aust. J. Chem. 21 (1968) 1683.

[118] Buck, U., Pauly, H., Z. Phys. 208 (1968) 390.

[119] Parson, J. M., LeE, Y. T., Entropie 42 (1971) 146.

[120] Chen, C. H., Siska, P. E., Lee, Y. T., J. Chem. Phys. 59 (1973) 601.

[121] Dunham, J. I., Phys. Rev. 41 (1932) 721.

[122] Bickes, R. W., Bernstein, R. B., Chem. Phys. Lett. 26 (1974) 457.

[123] Nain, V. P. S., Aziz, R. A., Jain, P. C., Saxena, S. C., J. Chem. Phys. 65 (1976) 3243. 